\title{
Copyright
}

by

Urairisa Birdy Phathanapirom

2014 
The Thesis Committee for Urairisa Birdy Phathanapirom Certifies that this is the approved version of the following thesis:

\title{
Nuclear Fuel Cycle Transition Analysis Under Uncertainty
}

\section{APPROVED BY}

\section{SUPERVISING COMMITTEE:}

Supervisor:

\author{
Erich A. Schneider
}

Jay W. Zarnikau 


\title{
Nuclear Fuel Cycle Transition Analysis Under Uncertainty
}

\author{
BY \\ Urairisa Birdy Phathanapirom, B.S. Physics
}

Thesis

Presented to the Faculty of the Graduate School of

The University of Texas at Austin

in Partial Fulfillment

of the Requirements

for the Degree of

Master of Science in Engineering

The University of Texas at Austin

August 2014 
philia 


\section{ACKNOWLEDGEMENTS}

I would like to thank the faculty in the Nuclear and Radiation Engineering Program. First, thank you to my research advisor, Erich Schneider. His drive, support, and encouragement have helped me achieve more than I thought possible. Second, thank you to Steven Biegalski for first opening the doors to me as an undergraduate researcher. And a special thank you must go to Sheldon Landsberger, for making me take the steps I was too scared to take.

This research was performed using funds from the Nuclear Energy University Program's Graduate Fellowship, which is sponsored by the U.S. Department of Energy.

Thank you to my little family, especially Mrs. Butterscotch for being my faithful companion. 


\begin{abstract}
Nuclear Fuel Cycle Transition Analysis Under Uncertainty

Urairisa Birdy Phathanapirom, M.S.E.

The University of Texas at Austin, 2014
\end{abstract}

Supervisor: Erich A. Schneider

Uncertainty surrounds the future evolution of key factors affecting the attractiveness of various nuclear fuel cycles, rendering the concept of a unique optimal fuel cycle transition strategy invalid. This work applies decision-making under uncertainty to fuel cycle transition analysis, demonstrating a new, systematic methodology for choosing flexible, adaptable hedging strategies that yield middle-of-the-road results until uncertainties are resolved.

A case study involving transition from the current once-through light water reactor (LWR) fuel cycle to one relying on continuous recycle in fast reactors (FRs) is cast as a no-data decision problem. The transition is subject to uncertainty in the cost of spent nuclear fuel (SNF) and high-level waste (HLW) disposal in a geologic repository, slated to open some years into the future. Following the repository open date, the cost of SNF and HLW disposal is made known, and may take on one of five possible values.

Strategies for the transition are enumerated and simulated using VEGAS, a systems model of the nuclear fuel cycle that solves for its material balance and applies input cost data to calculate the associated annual levelized cost of electricity (LCOE). Perfect 
information strategies are found using the lowest average, maximum, and integrated LCOE objective functions. The loss in savings for following a strategy other than the perfect information strategy is the "regret" which is calculated by evaluating the performance of each strategy for every end-state. Hedging strategies are then selected by either minimizing the maximum or the expected regret.

Generally, the optimal hedging strategy identified using the decision methodology suggests a partial transition to a closed fuel cycle prior to the repository open date. Once the repository opens, the transition may be abandoned or accelerated depending on which disposal cost outcome is realized. The lowest average and integrated LCOE objective functions perform similarly; however, the lowest maximum LCOE objective function appears overly sensitive to aberrations in the annual LCOE that arise due to idle reprocessing capacity. The minimax regret choice criterion is shown to be more conservative than the lowest expected regret choice criterion, as it acts to hedge against the worst-case outcome.

By following a hedging strategy, agents may alter their fuel cycle strategy more readily once uncertainties are resolved. This results since hedging strategies provide flexibility in the nuclear fuel cycle, preserving what options exist. To this end, the work presented here may provide guidance for agent-based, behavioral modeling in fuel cycle simulators, as well as decision-making in real world applications. 


\section{TABLE OF CONTENTS}

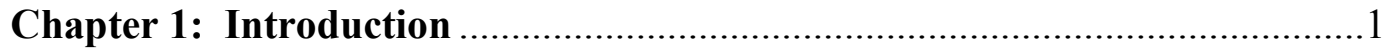

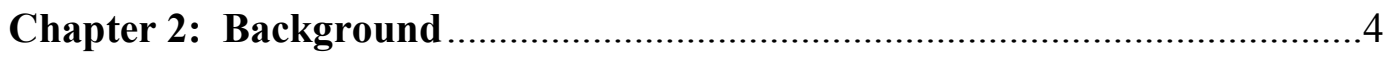

2.1 Decision Making with Imperfect Knowledge........................................4

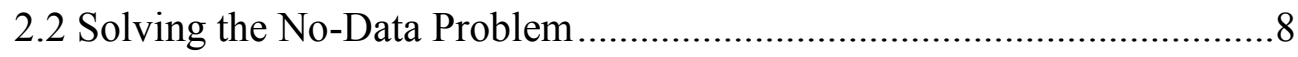

2.3 Decision Making in the Nuclear Fuel Cycle ........................................14

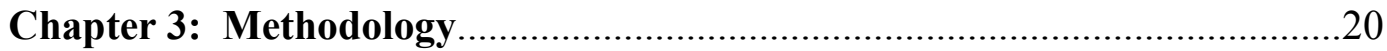

3.1 Imperfect Knowledge and the Nuclear Fuel Cycle .............................20

3.2 VEGAS Nuclear Fuel Cycle Simulator .............................................23

3.2.1 VEGAS Levelized Cost of Electricity Calculation...................27

3.3 Approach to the No-Data Problem .................................................... 31

3.3.1 Description of VEGAS Simulations ….....................................32

3.3.2 Transition Strategies Under Perfect Information .......................35

3.3.3 Hedging Strategies Under Imperfect Information ....................36

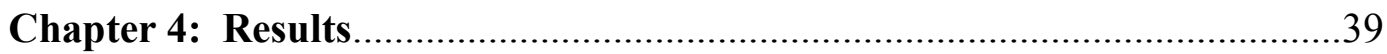

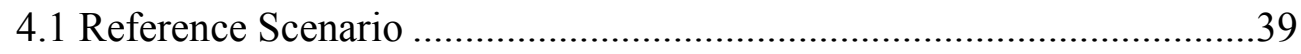

4.2 Sensitivity Study: Ratio Between HLW and SNF Disposal Costs .........51

4.3 Sensitivity Study: Fast Reactor Capital Cost ......................................58

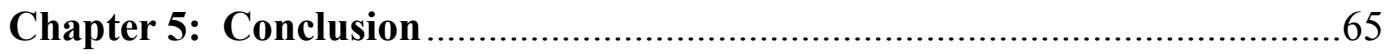

Appendix A: VEGAS Simulation Inputs ................................................6

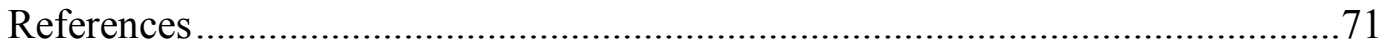

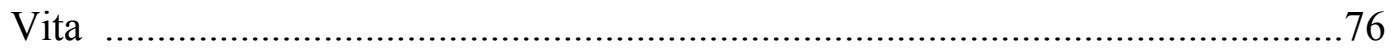




\section{Chapter 1: Introduction}

Uncertainty surrounds the evolution of key factors affecting the attractiveness of nuclear fuel cycle options. Large uncertainties in the cost, performance and even availability of advanced fuel cycle technologies may drive the timing of future choices and alter the available options and the criteria by which they are evaluated. Hence a single optimal strategy for transitioning from one nuclear fuel cycle to another does not exist.

Previous work in the area of fuel cycle transition analysis recognizes these uncertainties and call for "flexible" or "agile" fuel cycle options (Havlíček, 2008), (Piet et al., 2006). However, no clear methodology has emerged for selecting such a fuel cycle. Fuel cycle transition analyses have instead primarily employed the "scenarios" approach, which assumes that decision-makers would be able to correctly guess the future state of uncertain parameter(s). In effect, this approach ignores uncertainties or at best handles them in such a way that gives rise to inflexible courses of action.

The work presented in this thesis applies decision-making under uncertainty to fuel cycle transition analysis. Decision-making under uncertainty provides a systematic framework for choosing flexible transition strategies, termed "hedging" strategies, which yield middle-of-the-road results until uncertainties can be resolved. These hedging strategies are found using realistic depictions of uncertainty and limited information about the future, and are chosen in such a way as to reduce substantial losses if the state of nature that prevails is different than that which was expected.

A case study involving transition from the current once-through light water reactor (LWR) fuel cycle to one relying on continuous recycle in fast reactors (FRs) is cast as a no-data decision problem, in which it is impossible to gain information about the state of nature that will ultimately prevail. The transition is subject to uncertainty in the cost of 
spent nuclear fuel (SNF) and high-level waste (HLW) disposal in a geologic repository slated to open some years into the future. The cost of SNF and HLW disposal will be made known at the repository open date and may take on five possible states of nature.

Strategies for transitioning toward continuous recycle in FRs are enumerated using VEGAS, a systems model of the nuclear fuel cycle that solves for its material balance. VEGAS builds FRs at the maximum rate allowable based on existing LWR used fuel (UF) ${ }^{1}$ reprocessing capacity, and so a simulation is fully specified by its reprocessing capacity expansion profile. By applying input cost data to the material balance, VEGAS calculates the annual levelized cost of electricity (LCOE) for various reprocessing capacity expansion profiles. Using three different objective functions (the lowest average, maximum and integrated LCOE), perfect information strategies are determined. This is the so-called scenarios approach, which assumes that the end-state realized after the repository open date is known, and agents may choose to transition the fuel cycle in an optimal way based on the chosen objective function. The methodology given in this thesis instead evaluates the performance of each reprocessing capacity expansion profile for every possible end-state. The profile that performs best on the average for each end-state based on two different choice criteria (the minimax and expected regret) are then the chosen hedging strategy.

By following a hedging strategy, agents may alter their fuel cycle strategy more readily once uncertainties are resolved. This results since hedging strategies provide flexibility in the nuclear fuel cycle, preserving what options exist in the nuclear fuel cycle. Said differently, the "best" option may be the option that avoids foreclosing other options (Hamilton et al., 2012). To this end, the work presented here may provide guidance for

\footnotetext{
1 "Used fuel" is another term for "spent fuel". The difference arises from a policy issue of whether to regard the material as a resource or a waste. In this thesis, used fuel is used when referring to reprocessing/recycling of material, whereas SNF is used when referring to disposal of material.
} 
agent-based, behavioral modeling in fuel cycle simulators, as well as decision-making in real world applications.

Chapter 2 provides an introduction to the elements of decision theory utilized in this work, specifically defining key phrases such as imperfect knowledge and limited foresight. The no-data decision problem is described, as are techniques used for solving it. A review of nuclear-related studies that make use of decision analysis is also provided. Chapter 3 motivates and describes the transition scenario examined in this thesis. The VEGAS fuel cycle simulator tool used in the analysis is reviewed, and documentation of major inputs is given in Appendix A. The methodology for selecting optimal transition and hedging strategies is also presented. Chapter 4 gives the results from the reference transition scenario, as well as sensitivity studies involving varying the ratio between HLW and SNF disposal costs and FR capital costs. Chapter 5 draws conclusions based on the work presented. 


\section{Chapter 2: Background}

The work presented in this thesis aims to identify optimal transition strategies for nuclear fuel cycles given realistic depictions of uncertainty and limited information about the future. Optimal strategies are identified using a framework provided by compiling decision theoretic tools and systems models of the nuclear fuel cycle. The combination of

decision theory and systems analysis has been termed decision analysis (Huang et al., 1995). This work may then be viewed as an application of decision analysis. Early 1960s studies that incorporated decision analysis dealt with decision making in oil and gas exploration (Grayson, 1960), (Kaufman, 1963). Following these initial studies, decision analysis has reached many different applications due to advancement to different forms, each with a unique focus (Huang et al., 1995).

This chapter is divided into three sections. The first section serves to introduce the elements of decision theory utilized in this work, specifically defining key phrases such as imperfect knowledge and limited foresight, as well as provide an example of the no-data decision problem. The second section presents an overview of past techniques utilized in solving the no-data decision problem. The third section reviews past nuclear-related studies that utilize decision analysis.

\subsection{DECISION MAKING WITH IMPERFECT KNOWLEDGE}

Modern decision theory provides a systematic approach to choosing between alternative courses of action under conditions of imperfect knowledge. (Nykvist, 2005) ${ }^{2}$ identifies two key components to knowledge: information and foresight. Information refers to knowledge of how key future events will unfold, e.g. if a $\mathrm{CO}_{2}$ emissions cap will be

\footnotetext{
2 Some citations use the spelling "Nyqvist".
} 
imposed, whereas foresight refers to knowledge of how current actions or investments will affect the future, e.g. if building a nuclear instead of coal-fired power plant will later result in savings of $x$ or $y$ dollars. Imperfect knowledge may refer to either, or both, of these components, see Fig. 2.1. Agents who act with imperfect information, referred to as conditions of uncertainty, may be unaware of the level at which $\mathrm{CO}_{2}$ emissions will be capped. Likewise, agents acting with imperfect foresight, more commonly referred to as limited foresight, may choose to forego building a nuclear power plant and choose instead the coal fired plant because it is cheaper in the short-term.

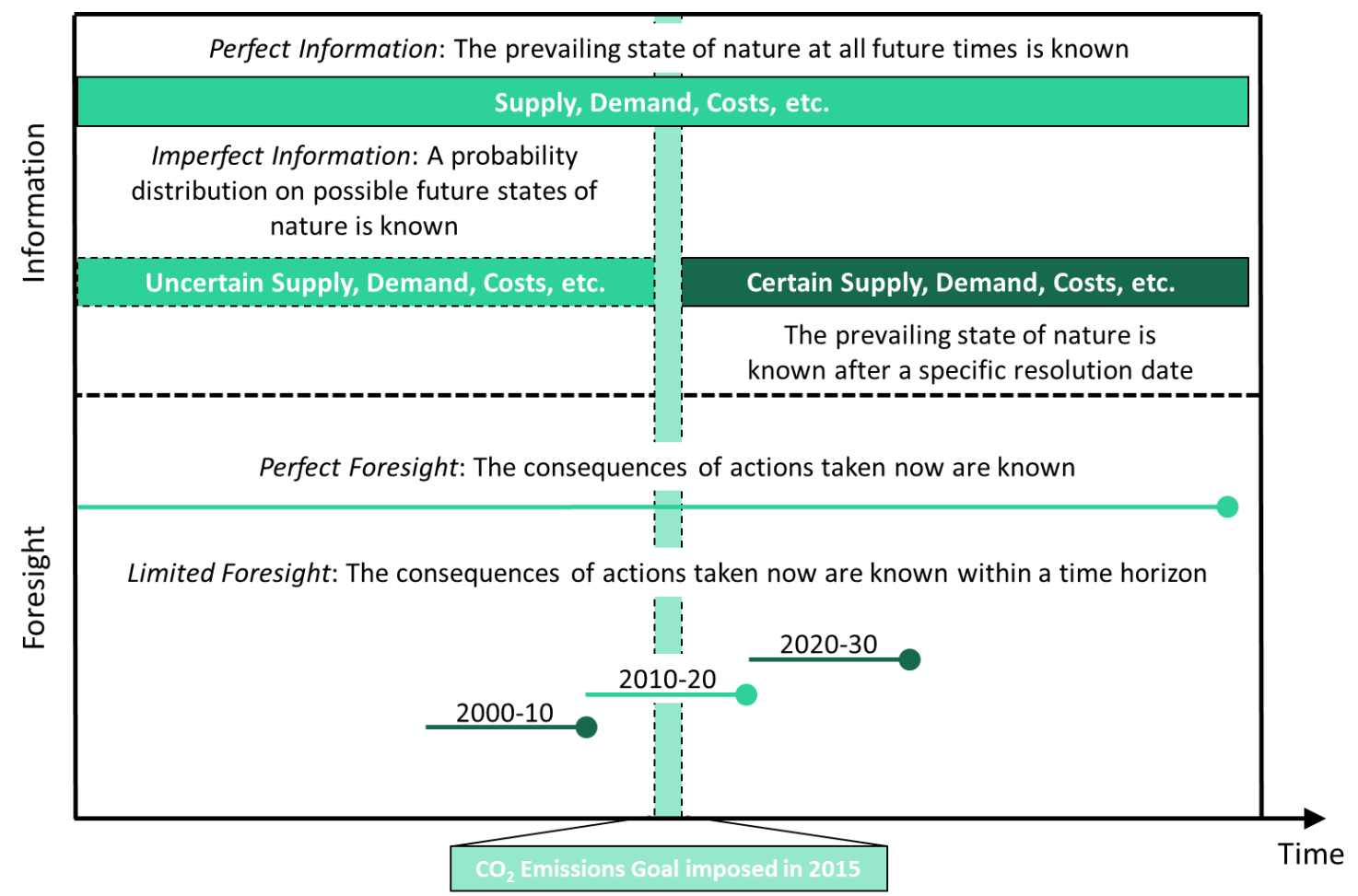

Fig. 2.1. Components of knowledge and their limiting cases.

Many energy system models are formulated in the limiting case of perfect knowledge (Azar et al., 2003), (EIA, 2009), (IEA, 2013), (Loulou et al., 2004). Under these conditions, producers and consumers optimize the energy system (typically through global cost minimization) over the simulation time period with perfect foresight and information 
of future economic and technological developments. The scenario created by these models should not be considered a forecast of the future, but rather a best-case scenario description of future developments, meant to provide guidance about how to achieve the best-case. In the other extreme, energy system models may operate under conditions of myopia, where short-sighted producers and consumers optimize their welfare in each time step of the simulation with no regard for how decisions made now will affect their future (Keppo et al., 2009), (Martinsen et al., 2006), (Nykvist, 2005). Completely myopic agents may be viewed as an extreme case of limited foresight, where the planning horizon is bounded by the coarsest time step available in the model. In this way, limited foresight actually imposes conditions of imperfect information on the system. In reality, decision makers act under conditions of imperfect knowledge in the domain between the extremes of perfect knowledge and complete myopia.

The work presented in this thesis assumes conditions of imperfect information, similar to the no-data decision problem found in decision theory. The no-data problem is one in which no experiment is readily available as a way of gaining information about the state of nature that will prevail. These problems consist of four components: (1) the available actions that can be taken, (2) the states of nature (or end-states) which may occur, (3) the consequences of each combination of action and state of nature (known as a stateact pair), and (4) a choice criterion by which the decision maker solves the final problem of choice.

The Wet or Dry problem is described here as an illustration of the no-data problem, adapted from the original presented in (Halter and Dean, 1971). Each year, California Thompson Seedless grape growers must choose how to produce and market their grapes. Thompson Seedless grapes are appropriate for either canning, fresh table consumption, wine production, or for sun-drying into raisins. The amount of land appropriated for 
canning grapes and table grapes must be assigned at the beginning of the season, but the remainder of the crop can be shifted late in the season to either wine grapes or to raisins. The industry commonly refers to this shift as "going wet or dry." The two available actions to a grower may be to allocate all of his acreage for raisins, or all of his acreage for wine crush:

$$
\begin{aligned}
& a_{1}=\text { allocate all acreage for raisins, or } \\
& a_{2}=\text { allocate all acreage for wine crush. }
\end{aligned}
$$

The consequences of the grower's action depend primarily on weather conditions and prices. At the time the grower chooses an action, he knows neither of these conditions, and he has no way of forecasting prices or of forecasting the weather. Raisins are sun-dried completely in the open, and rain during the drying period can inflict heavy losses on the grower who is going dry. Similarly, for the grower who is going wet, grapes remain on the vines several weeks longer than their dried counterpart, and there is very little chance of going dry after waiting for that period. The states of nature that may occur are all combinations of both weather conditions (rain or no rain) and relative prices (prices favor raisins or prices favor wine crush):

$$
\begin{aligned}
& \theta_{1}=\text { prices favor raisins; no rain, } \\
& \theta_{2}=\text { prices favor raisins; rain, } \\
& \theta_{3}=\text { prices favor wine crush; no rain, and } \\
& \theta_{4}=\text { prices favor wine crush; rain. }
\end{aligned}
$$

The choice criterion for the Wet or Dry problem is chosen as dollars profit per acre. The profits table for this problem is shown in Table 2.1. Each cell entry is determined by budgeting analysis utilizing the relevant prices, damage factors, and costs for the associated action and state of nature. The grower assigns probabilities $P\left(\theta_{i}\right)$ to the various states of nature based on past experiences. Given these probability assignments, the grower can calculate the expected value of each action. His strategy would then be to allocate his entire 
crop to raisins, or "go dry". Of course, a different set of probabilities could lead to a different action as optimal.

Table 2.1. Profits table for Wet or Dry problem.

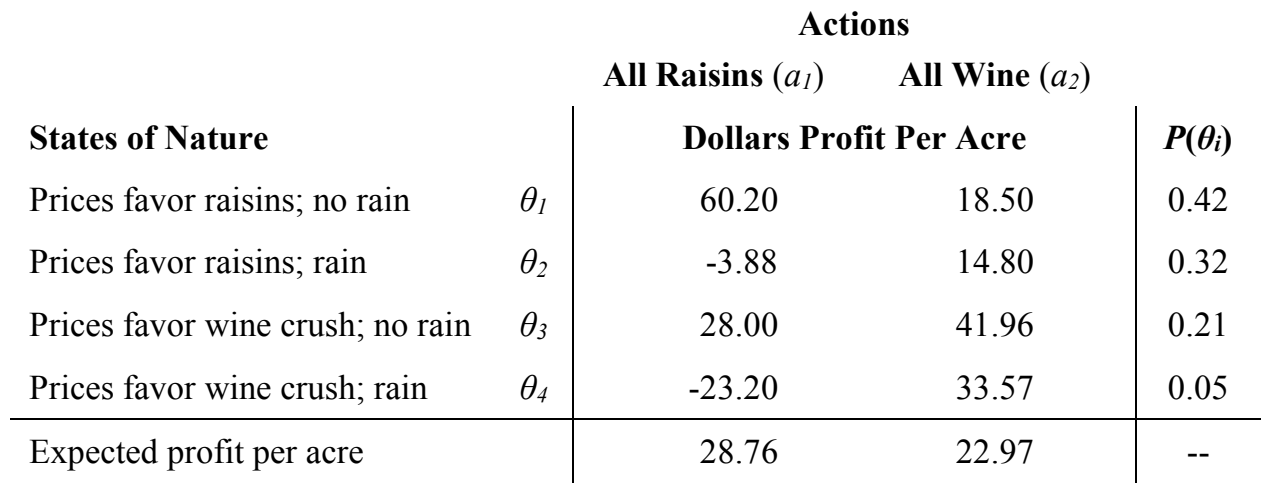

\subsection{Solving The No-Data Problem}

Several techniques have been proposed to address the no-data problem. These techniques are summarized in (Gorenstin et al., 1993) and are described for the two-stage problem, in which there is one state of nature for the first stage and two possible states of nature for the second stage; however, they may generalize to problems with a larger number of stages and states of nature. In this way, agents using the techniques described here act with perfect foresight but limited information.

1. Deterministic equivalent: In stochastic programming, the Deterministic Equivalent Problem is formulated by replacing random variables (all possible states of nature) with their expected values (Birge and Louveaux, 2011). The first-stage decisions are made based on the expected prevailing state of nature and represent the expected value solution. In the second stage, when the prevailing state of nature is realized, the secondstage decision must be taken.

(Nykvist, 2005) examines the effect of limited foresight on the global energy system model GET (Azar et al., 2003). In its original formulation, the GET model solves 
for a globally aggregated fuel mix across three end-use energy sectors which provides the lowest energy system cost while meeting a $\mathrm{CO}_{2}$ emissions cap in the year 2100. Nykvist imposes limited foresight by dividing the simulation time period into $T$ time steps, and for each time step $\tau$ a local optimal solution is found. For the following time step $t_{\tau+1}$, the initial state is defined by the results of the previously executed optimization. By implementing limited foresight in this way, the $\mathrm{CO}_{2}$ emissions goal of 2100 is not visible at the beginning of the GET simulation and has no effect on the decision making. With this formulation of GET, Nykvist demonstrates that the costs of realizing a $\mathrm{CO}_{2}$ target of year 2100 increase with shorter allotted foresight.

Using a similar formulation, (Leibowicz et al., 2012) develops a two-stage framework for the energy-economic model MARKAL (Loulou et al., 2004) to examine effects of uncertainty ${ }^{3}$ in nuclear electricity costs on the optimal electricity generation technology mix. A standard MARKAL run configures the energy system in order to minimize the net total cost of the system with full knowledge of future events. In the twostage framework, MARKAL is run over the entire time horizon without any change in costs in the year 2030 (base run); following, MARKAL is re-run assuming a change in nuclear electricity costs beginning in year 2030, using results from the base run prior to year 2030 . Leibowicz concludes that carbon mitigation not heavily reliant on nuclear power is economically inefficient.

The primary issue with the deterministic equivalent approach is that the optimal plan in the first stage is only optimal under the assumption that the future conditions will occur as expected. For example, the limited foresight formulation of MARKAL, SAGE (Loulou et al., 2004) will not properly respond to a constraint representing the total reserves

\footnotetext{
${ }^{3}$ (Leibowicz et al., 2012) actually claims to examine the effect of limited foresight on the optimal electricity generation technology mix. However, for consistency with Section 2.1, this has been changed to uncertainty.
} 
of a resource such as gas or coal if the constraint doesn't come into effect within the relevant time horizon. SAGE instead uses as much gas or coal as needed in each period, without any planning ahead for this eventuality, until the period when the reserve is exhausted.

2. Scenarios: The optimal plan is found for all $N$ possible scenarios, obtaining a set of $N$ solutions. The number of scenarios is determined by taking all available combinations of possible states of nature. In this way, the scenarios approach assumes perfect information by assuming that $N$ decision-makers would be able to guess the correct state of nature that will prevail. A single optimal plan is constructed by observing similarities and trends in the $N$ set of solutions which can be expected to perform well under all scenarios.

(Draper, 2001) presents a limited foresight variant of the CALVIN model (Lund et al., 1999), an optimization model of California's inter-tied water system. Draper imposes limited foresight in the CALVIN model in a similar manner to Nykvist in the GET model. In the CALVIN model, historical hydrologic data is exogenously applied and economic drivers are used to allocate water from California's surface and ground water supplies to minimize the water shortage cost. This hydrologic data set is chosen due to its availability and supposed representation of the range of possible flows and their implicit spatial and temporal correlation structure. This range may be viewed as offering different scenarios of hydrologic conditions, and reservoir operating rules are then derived using "a mixture of statistical tools and engineering judgment" from the model output.

The limitation of the scenarios approach is the difficulty of constructing a single strategy which is adequate "on the average" for all scenarios. For example, if one were to optimize the energy generation technology mix for two differing energy demand growth scenarios, the two scenarios will produce very different recommendations on technology investments. Typically, there are no easy guidelines for constructing a strategy that is 
adequate on the average for both scenarios. The only exception are "robust" strategy components that are part of the optimal strategy in both scenarios. Indeed, some studies simply present optimal strategies for different scenarios, with no effort made to aggregate the strategies (IEA, 2013), (Leibowicz et al., 2012).

(Rockafellar and Wets, 1991) investigate this problem in the abstract, formulating a "Progressive Hedging Algorithm" that averages solutions from the scenarios approach to determine a solution which is in some sense good for the underlying problem. The algorithm's fundamental idea is to add the only constraints that tie together the different scenarios to the objective function via Lagrange multipliers, creating a function that may be decomposed. While the algorithm has been successfully applied to various topics such as market production planning and network flow problems (Escudero et al., 1993), (Mulvey and Vladimirou, 1991), it is not applicable in the case of nuclear fuel cycle simulators. The algorithm is constructed for a stochastic linear program (LP), whereas fuel cycle simulators are formulated as systems tools that track resource flows.

3. Stochastic optimization: Stochastic programming is a means to remedying the weaknesses of the scenarios approach; it determines a unique strategy which is optimal "on the average" for all scenarios by explicitly embedding uncertainties as future bifurcations with assigned probabilities within a single coherent formulation, termed a stochastic model.

(Loulou et al., 2004) presents a stochastic programming variant of the standard MARKAL model. In this model, multiple scenarios are represented within the stochastic model. The example given by Loulou concerns the greenhouse gas (GHG) emissions from the Quebec energy system, which is subject to an upper bound on its cumulative GHG emissions over the time period 2000 to 2030. However, the upper bound may take on any of four values, each with a specific probability of occurrence, and is resolved in 2015. The optimal hedging strategy is then found based on the expected cost choice criterion. This 
strategy takes into account that any of the four possible states of nature may occur, and chooses a middle-of-the-road path until the uncertainty is resolved, see Fig. 2.2, states of nature 1-4. This is in contrast to the scenarios approach, in which there would exist four optimal strategies from 2000 to 2015 , none of which may match the optimal hedging strategy, see Fig. 2.2, scenarios 1-4. Loulou does mention that the commonly used expected cost choice criterion presupposes that probabilities of event outcomes are available and that the decision maker is risk neutral; both of these assumptions are debatable. Risk, sometimes termed regret, refers to the difference between the largest gain and that which the decision maker receives. One practical limitation of the stochastic programming approach is the growth in model size as the number of event bifurcations increases, which may prove impractical with finite computing power.

(Gilbert and Richels, 1981) estimate the expected economic value of the United States (U.S.) Department of Energy (DOE) National Uranium Resource Evaluation Program (NURE) using ETA-MACRO, a nonlinear program that allows for energyeconomy interactions, cost-effective conservation of energy, interfuel substitution, and new energy supply technologies (Manne, 1979). The main output of the NURE program is information about the distribution of uranium resources at the cost of several million dollars. The net benefit of this information is estimated using a stochastic model of future nuclear power development that allows for five policy decisions: parallel development of both a fast breeder reactor (FBR) and an advanced converter reactor (ACR), development of just one type, wait, or stop (see Fig 2.3). After an initial decision is made, a filtration of future decisions occurs (example: if a FBR demonstration plant is built today, a successor FBR, labelled CBR-1, may be built in 20 years, but not a successor ACR plant, labelled ACR-1). The ensuing decision tree, a model of decisions and their possible outcomes (see 
Fig. 2.3), is often associated with Real Options Valuation or Real Options Analysis (ROV or ROA), which extends from decision making under uncertainty.

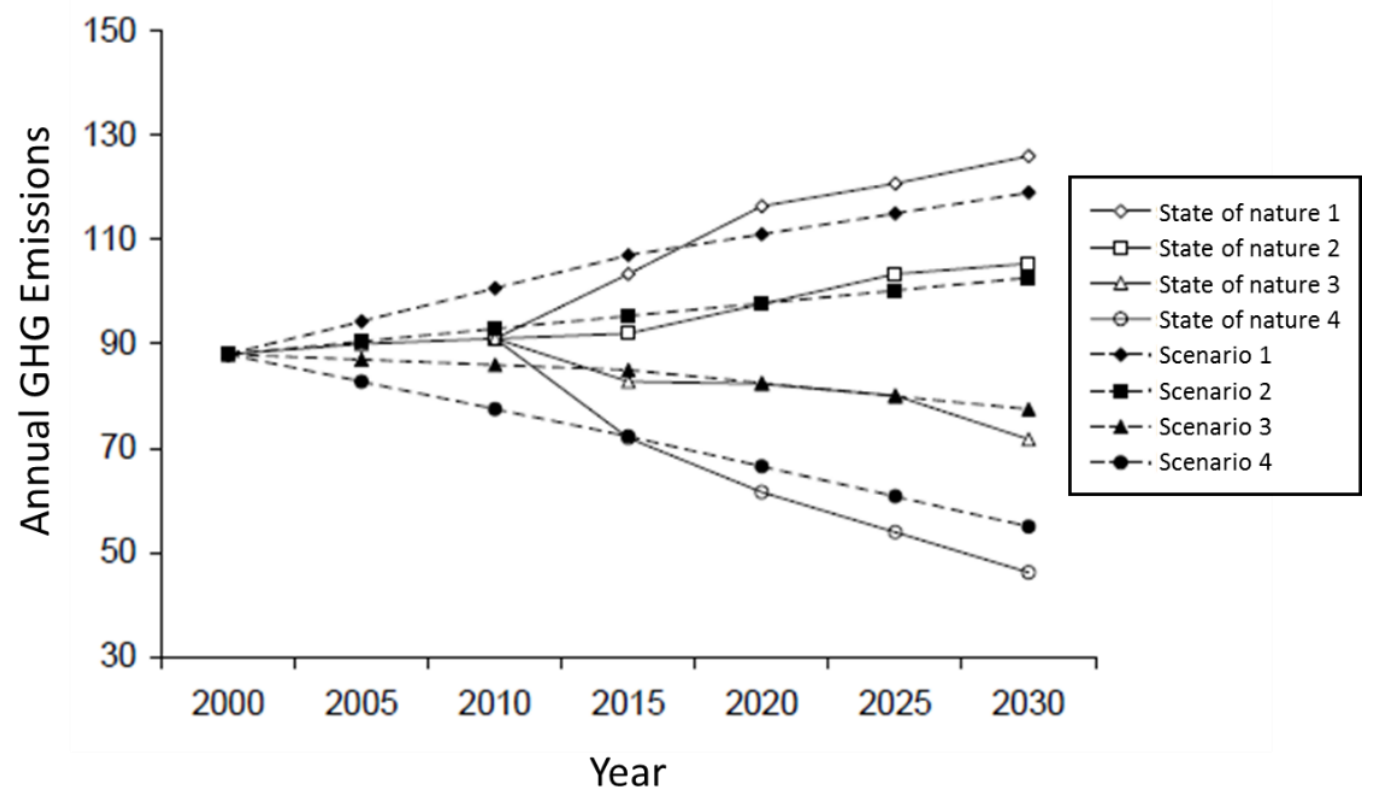

Fig. 2.2. Optimal GHG trajectories found under stochastic model (states of nature 1-4) and scenario analysis (scenarios 1-4). Adapted from: (Loulou et al., 2004)

The ROA approach allows for flexibility in decision making and is largely applied to the analysis of investment projects (Yao and Jaafari, 2003). Prior to ROA, valuation of projects was made based on a "go/no-go" decision approach to the entirety of a project at the time of project feasibility studies and approval. The ROA approach allows for real options or courses of action available to decision makers, which include deferring, scaling down, staging, or even abandoning projects in response to additional information, such as market conditions. In this way, proactive risk management is integrated into the analysis of investment projects. In the example provided by (Gilbert and Richels, 1981), options (enumerated in Fig. 2.3) include building demonstration FBR or ACR plants, and 
information of uranium resources provided through the NURE project influence which options are realized.

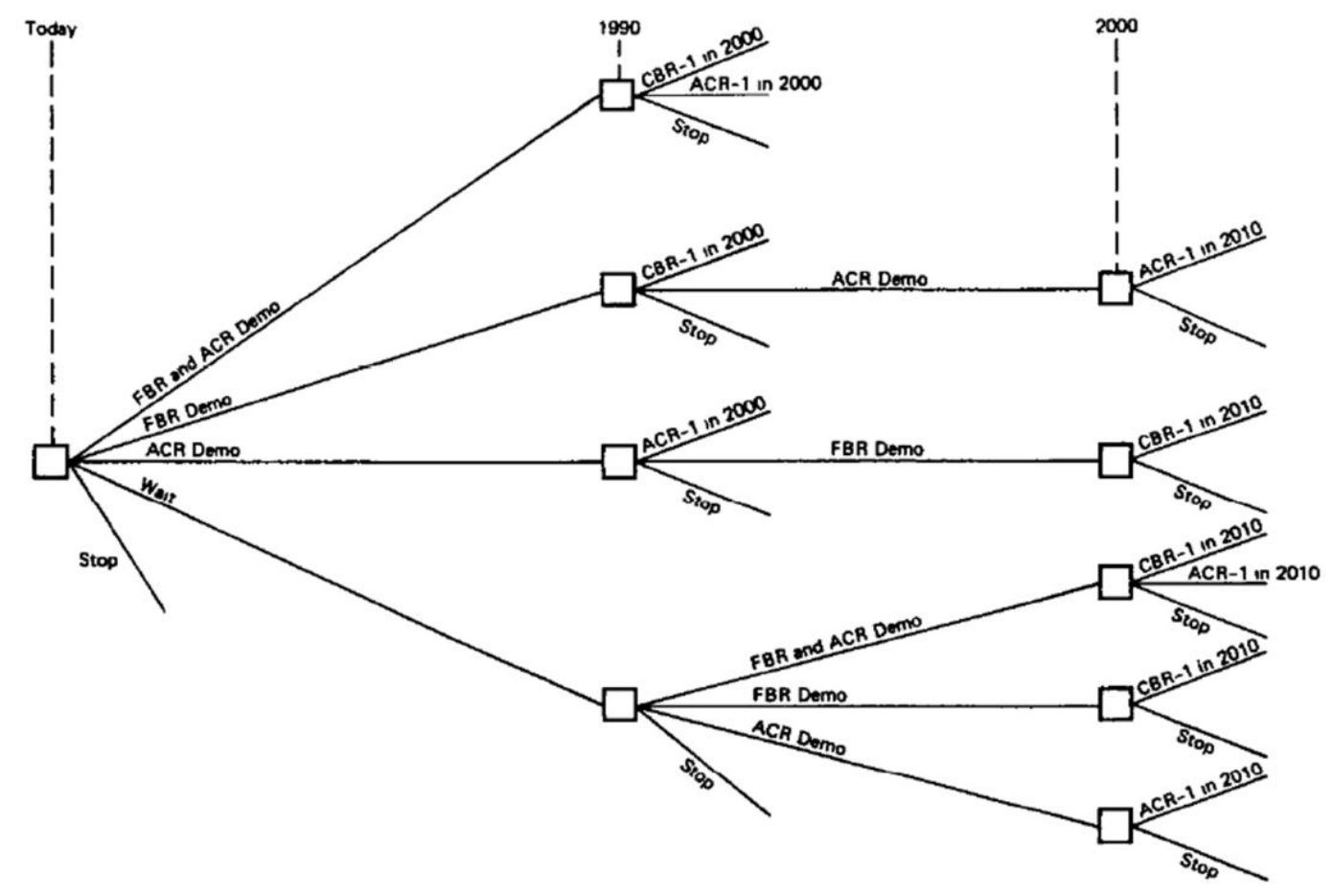

Fig. 2.3. Decision tree of alternative nuclear fuel cycles. Source: (Gilbert and Richels, 1981)

\subsection{DeCision MAKING IN THE NuCLEAR FUel CYCLE}

(Zhou et al., 2006) reviews 252 publications that use decision analysis in energy and environmental modeling. Of these publications, the share of nuclear-related studies has been as high as 30 percent (from 1975 to 1984), with a later decline to 7 percent (from 1995 to 2004), covering a wide variety of topics. A selection of examples are related below.

(Evans, 1984) applies probabilistic decision analysis utilizing a LP model (Evans, 1981) in order to estimate the likelihood of a net benefit from the Sizewell B pressurized water reactor project in the UK. In a scenarios-like approach, two runs of the LP are contrasted, one with and one without the Sizewell B project. Both LP runs strategically 
sample from the complete space of uncertain parameters in order to derive sensitivities in the overall outcomes.

The ROA approach is taken in (Rothwell, 2006) in order to estimate the net present value of building new nuclear power plants, specifically an Advanced Boiling Water Reactor in Texas. Uncertainties are modeled using Monte Carlo simulation, and various risk premiums (the minimum amount by which the expected return on a risky action must exceed the known return on a risk-free action in order for decision makers to select the risky action) are derived that would trigger investment in new nuclear power plants.

(Gregory and Lichtenstein, 1987) critique two reports concerning the site selection for characterization of a potential U.S. HLW repository. The first report, (DOE, 1986), evaluates five potential sites using a multiattribute utility analysis (MUA), designed to aid in the selection process. MUA explicitly considers multiple criteria in decision-making by weighting attributes based on relevance and constructed value functions for those attributes based on a prior distribution of preferences. The attributes included in (DOE, 1986) are worker and public fatalities; and aesthetic, archaeological, biological, and socioeconomic impacts. However, despite ranking the five potential sites based on these attributes, the second report, (DOE, 1986a) recommended three sites ranked first, third, and fifth in (DOE, 1986). Gregory and Lichtenstein point out a key issue surrounding MUA: some decision analysts state that decision aids like MUA should not dictate policy but should be viewed merely as providing information to the decision maker.

A quantitative model for decision making in nuclear safeguards based on a tradeoff between risk diversion and the cost of reducing the risk is presented in (Judd and Weissenberger, 1982). Uncertainties are accounted for in a diversion model (which enumerates the type of attempts to divert special nuclear material) and a consequence model (which gives values to consequences of successful diversion attempts), both of 
which are affected by the proposed safeguards technology. Then, if the expected value of consequences exceeds the cost of the safeguards technology, it is suggested that the safeguards technology is employed.

Fewer applications of decision analysis are found in the development of the nuclear fuel cycle. This is despite the number of uncertainties that exist regarding the nuclear fuel cycle (e.g. demand growth rates, economic costs, and uranium resource availability) and the systematic framework for handling these uncertainties which is offered by decision analysis. Nonetheless, three applications of decision analysis to the development of nuclear power are described below.

(Gilbert and Richels, 1981), discussed previously in Section 2.2, estimate the expected economic value of the U.S. DOE NURE program. Similarly, (Manne and Richels, 1978) utilize the ETA-MACRO program to evaluate alternative R\&D strategies regarding the U.S. breeder reactor program with respect to uranium supply, energy demand growth, environmental constraints on coal and shale oil production, and the availability of alternative energy sources. Individual respondents' subjective probabilities obtained through questionnaires are used for distributions on critical uncertainties. The authors found that, in general, a positive expected dollar benefit was predicted from any breeder development program, despite the possible risk of unnecessarily incurred research and development costs if breeder reactors are never brought into widespread commercial use.

(Kunsch and Teghem, 1987) take a MUA approach to the decision to reprocess SNF utilizing STRAtegy for the Nuclear Generation of Electricity (STRANGE), a stochastic LP (Teghem et al., 1986). The examined criteria include production costs, energy independence, commercial balance, and national technological level. An optimal strategy between reprocessing and disposing SNF is determined for each pair of objectives (either minimize NU needs or minimize costs) and scenarios. The "first compromise" is 
found between the two optimal strategies using the minimax principle (Wald, 1945), in which the maximum regret is minimized. The scope of the work presented is limited to fuel cycle policy in the short term (20-30 years).

(Pierpoint, 2011) presents a MUA for the selection of transition strategies for the nuclear fuel cycle. A 100 year time period is simulated using the Flexible Advanced Nuclear Technology Simulation by Year (FANTSY) model (written by the author) and a decision analytic model of the U.S. nuclear fuel cycle is applied following the simulation. In general, Pierpoint considers continuation of the once-through LWR fuel cycle versus gradual or rapid FR capacity expansion. A multiattribute utility function is created consisting of a cost metric (total system cost, discounted to the present year) and waste metric (SNF, transuranics (TRU), and fission product (FP) masses). The value of a scenario, consisting of a combination of decision values and uncertainties, is calculated following a FANTSY simulation. Optimal strategies are chosen through minimization of the multiattribute utility function following enumeration of all scenarios in FANTSY. Some conclusions drawn from Pierpoint's study may be influenced by the choice of decision criteria, specifically the cost metric. Pierpoint indicates that partial FR builds early in the simulation and full FR builds late in the simulation are preferred due to steep discounting of costs for reactors built later in the simulation. Infinite capacity of supporting fuel cycle technologies are also assumed, which may lead to idle capacity following initial start-up in a real world situation.

Earlier fuel cycle analysis methodologies have taken the "scenarios" approach, discussed in Section 2.2. While the scenarios approach may provide insight into how the fuel cycle may evolve over time, it is constrained by the modelers' judgment. The scenarios modeled are often limited in number and reflect the states of nature which the modeler believes within reason may occur. With the scenarios approach, there is no systematic 
approach to varying scenario parameters in an attempt to seek the "best" option for fuel cycle development.

(Yacout et al., 2004) examines maintaining the level of nuclear capacity throughout the century under four different scenarios: once-through LWR with spent fuel separation only, single mixed-oxide (MOX) recycle, and single and double tier transmutation systems. The single tier system involves recycling into MOX fuel for advanced LWRs, whereas the double tier system includes an initial MOX fuel pass, followed by continuous transuranic recycle in FRs. Scenarios are further enumerated through a range of reprocessing capacities and inclusion of a modest-growth scenario (1.5 percent), and are modeled using DYMOND (DOE, 2002) and DANESS (Van Den Durpel et al., 2003), and verified by NFCSIM (Schneider et al., 2005). Impacts on repository performance for each scenario are examined through assessment of the waste heat load. Similarly, the Dynamic Systems Analysis Report for Nuclear Fuel Recycle (DSARR) (Dixon et al., 2008), examines systems costs, uranium resource impacts, and waste management impacts involved in transitioning fuel cycles from the current once-through. Scenarios examined include once-through; single tier recycling in FRs only; and two tier recycling, first in LWRs, and then in FRs, using VISION (Yacout et al., 2006). Impacts due to variations in reprocessing capacity deployed and rate of deployment, FR conversion ratio, and demand growth are investigated for each scenario.

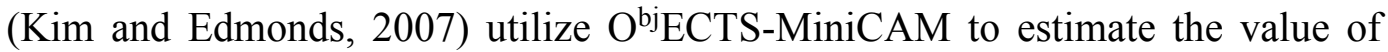
nuclear energy for future $\mathrm{CO}_{2}$ mitigation. $\mathrm{O}^{\text {bj ECTS-MiniCAM}}{ }^{4}$ is a long-term, global integrated assessment model of energy-economic interactions and GHG emissions (Kim et

\footnotetext{
${ }^{4}$ The Object-oriented Energy, Climate, and Technology Systems ( ${ }^{\text {bjeCTS }}$ ) framework is key to allowing expansion of nuclear fuel cycle simulation capabilities in the $\mathrm{O}^{\text {bj }}$ ECTS-MiniCAM model. The $\mathrm{O}^{\text {bj }} \mathrm{ECTS}$ framework allows for modular integrated assessment modeling, similar to the Cyclus nuclear fuel cycle simulator project (Wilson et al., 2012).
} 
al., 2006). While the energy-economic interactions operate similarly to MARKAL,

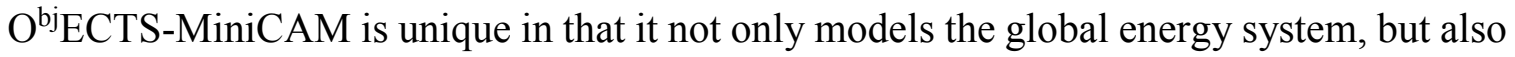
the nuclear fuel cycle. Kim and Edmonds have extended the fuel cycle simulation capabilities of $\mathrm{O}^{\mathrm{bj}} \mathrm{ECTS}-\mathrm{MiniCAM}$ in order to track waste production and composition, reprocessing, and subsequent reuse or disposal of UF components. Kim and Edmonds evaluate two groups of nuclear power technologies: currently operating LWRs and advanced LWRs (ALWRs), both assumed to utilize a once-through fuel cycle, despite reprocessing capabilities available in the model. A reference long-term energy demand trajectory is constructed, and several emissions constraints (including zero constraint) that stabilize the atmospheric $\mathrm{CO}_{2}$ concentration with and without new nuclear deployment are applied. Comparison of these cases allows estimation of the value of nuclear energy for mitigating $\mathrm{CO}_{2}$ emissions, as well as exploration of the challenges associated with large scale deployment of nuclear power in an emissions constrained world.

The work presented in this thesis offers a new perspective on handling uncertainty in analyses regarding the nuclear fuel cycle. This perspective is informed by the field of decision theory, namely its applications of decision making under uncertainty and ROA. In general, decision making has never been handled in this way in fuel cycle transition analysis. While (Pierpoint, 2011) does attempt to confront the challenge of decision making under uncertainty, the approach is largely different than that taken here. A methodology is presented for selecting hedging strategies for fuel cycle expansion when uncertainties exist in fuel cycle parameters. These hedging strategies take a "middle-of-the-road" path until either more information may be known, or the uncertainties are resolved. This work is intended to lay the foundations for implementing autonomous, dynamic decision making within fuel cycle simulators. 


\section{Chapter 3: Methodology}

In this chapter, motivation is given for the transition scenario examined in this work. First, the scenario is described and cast as a no-data decision problem. The basic functionalities and inputs to the VEGAS fuel cycle simulator is described. Appendix A provides more detailed documentation of inputs to the VEGAS tool for the transition scenario examined here. Finally, a methodology for selecting the optimal transition and hedging strategies is presented.

\subsection{IMPERFECT KNOWLEDGE AND THE NUCLEAR FUEL CYCLE}

The transition scenario studied in this work is motivated by the Spent Nuclear Fuel Recycling Program Plan report to Congress (DOE, 2006). This report describes the SNF Recycle System initially identified by DOE as a pathway to incorporating continuous fuel recycling into the U.S. nuclear fuel cycle in order to recover more energy and reduce waste, depicted in Fig. 3.1. In the SNF Recycle System, LWR SNF from past and existing LWRs is separated into FPs for storage and subsequent disposal, uranium for reuse or storage, and TRU elements. TRU elements are then burned in FRs, producing electricity while achieving partial TRU destruction. FR spent fuel is subsequently separated into the same

three streams: U, FPs, and TRU. The separated TRU is then recycled and again burned in FRs. This closed fuel cycle is aimed at fissioning the initial TRU inventory in legacy SNF while also accepting new TRU from the LWR SNF. More details of the SNF Recycle System are provided in (DOE, 2006).

One key source of uncertainty regarding the proposed fuel cycle in (DOE, 2006) is the cost of SNF and HLW disposal in a geologic repository (Dixon et al., 2008). Given this uncertainty, the potential benefits of a transition from the current once-through LWR cycle to one similar to the SNF Recycle System are unclear. If the cost of disposal proves to be 
inexpensive, the transition could be delayed or even abandoned at the expense of requiring more repository space. Alternatively, if the cost of disposal proves high, it would likely be optimal to pursue an aggressive schedule for closing the fuel cycle to ensure that enough reprocessing and FR capacity can be installed to minimize the need for repository space.

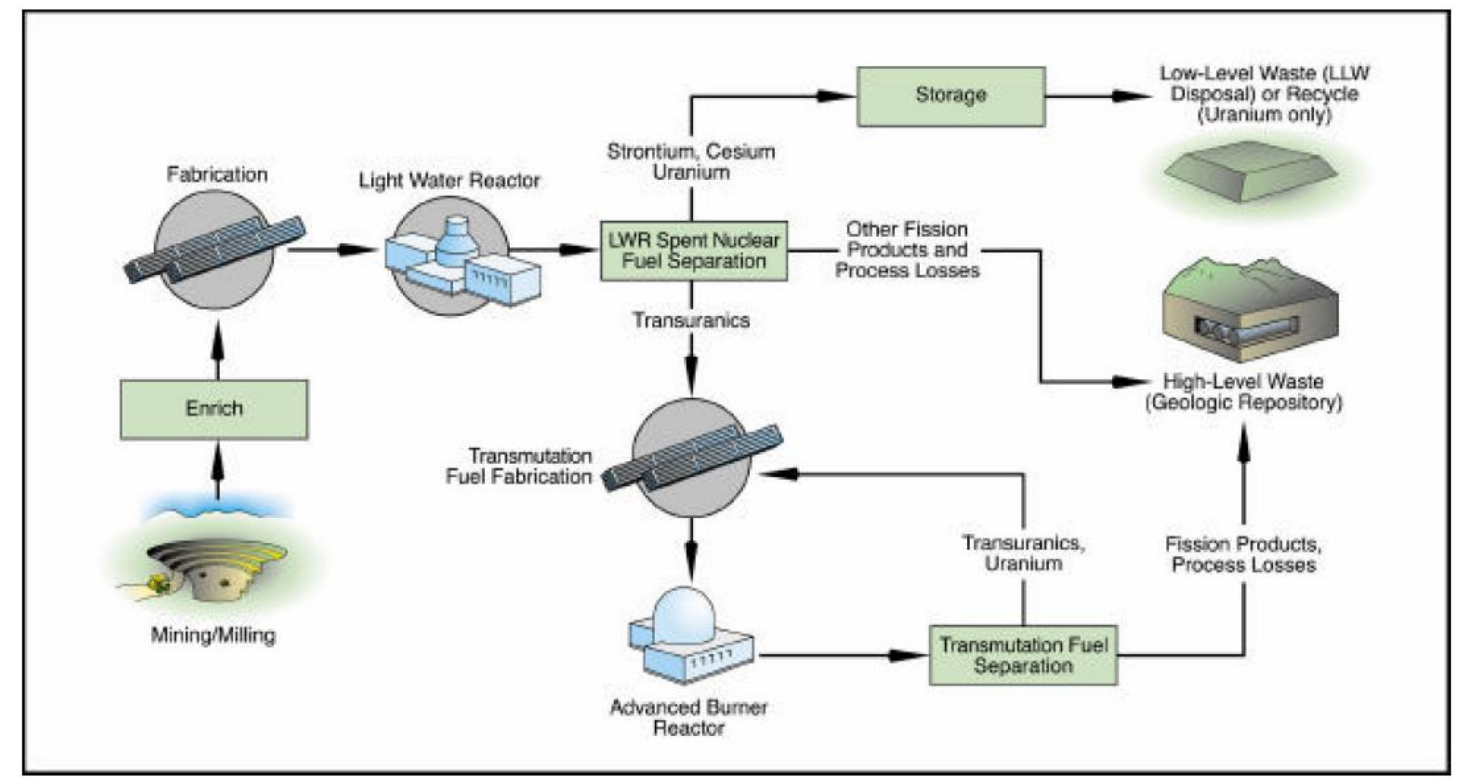

Fig. 3.1. Schematic of SNF Recycle System. Source: (DOE, 2006)

The scenario examined in this work is described in the following text. Simulating transition strategies between once-through and continuous FR recycle while incorporating the uncertainty in SNF and HLW disposal costs gives rise to a no-data decision problem. Each stage is defined as the time interval between bifurcation points. The simulation time period, from 2015 to 2100 , is divided by three bifurcation points, creating four discrete stages (Fig. 3.2). A repository is slated to open in the year 2070, whose operating costs may take on five different end-states.

At the beginning of the simulation in year 2015 , the current $100 \mathrm{GW}_{\mathrm{e}}$ fleet of LWRs is operating on the traditional once-through fuel cycle. The demand growth rate for nuclear electricity is 2 percent per year. Leading up to year 2030, two events occur which may alter 
the existing fuel cycle: (1) UF reprocessing technology becomes commercially available, and (2) an independent federal authority is appointed for implementing a nuclear waste management program. The introduction of an independent federal authority is supported by both public opinion (NEI, 2013) and the American Nuclear Society (ANS, 2009). Creation of an independent authority is expected to aid in "scientifically sound and technically informed decision making in the U.S. nuclear fuel cycle policy," specifically allowing for long-range planning (ANS, 2009). If such an authority were to materialize, it may be reasonable that it would advocate a closed fuel cycle to achieve better uranium resource and repository space utilization.

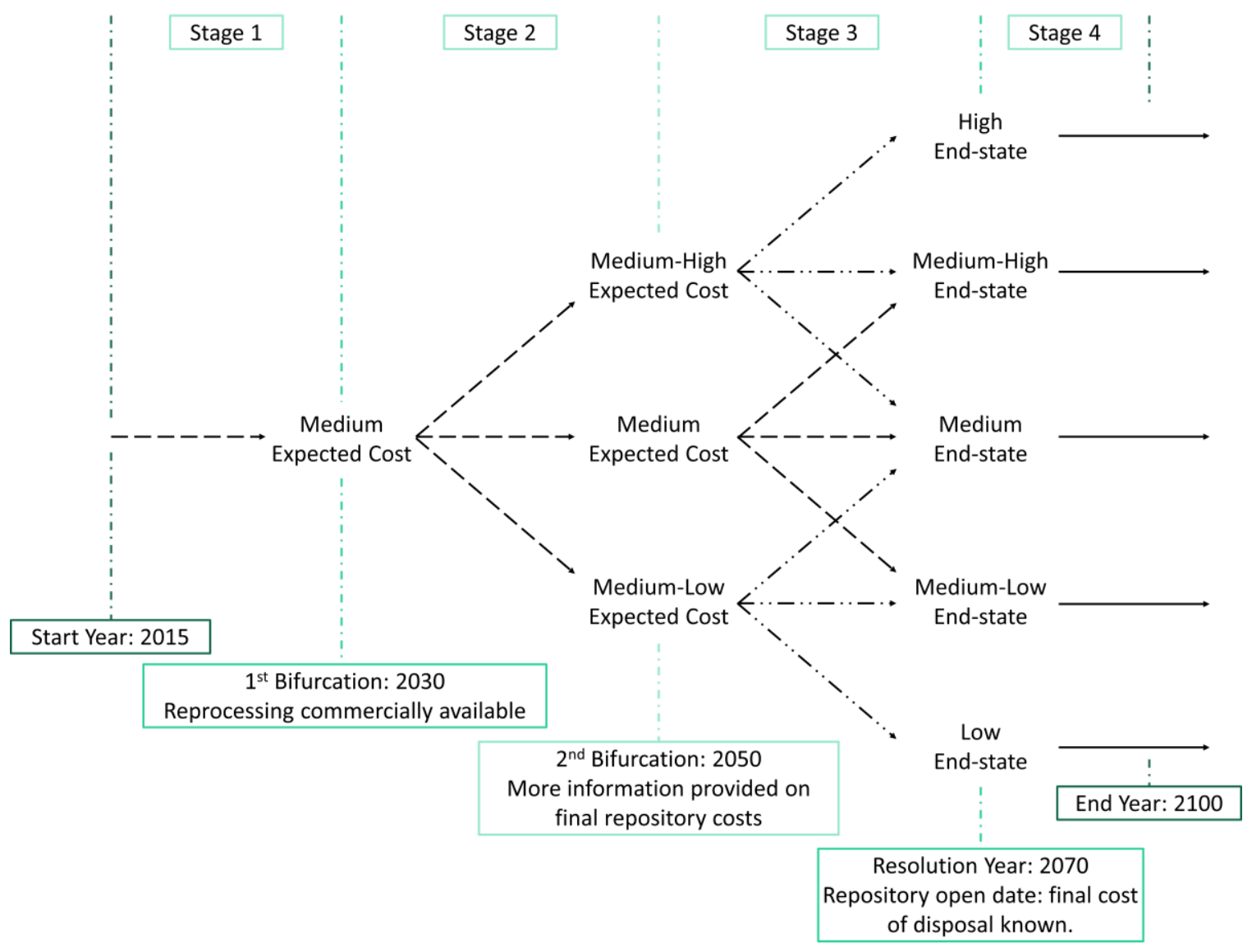

Fig. 3.2. Evolution of information on SNF and HLW disposal costs. Dashed lines indicate cost outcome uncertainty. 
The uncertainty in the final SNF and HLW disposal costs for a geologic repository is depicted in Fig. 3.2. This uncertainty is the greatest at the beginning of the simulation, in stages 1 and 2, when all end-states are viewed as possible. Decisions made through the end of stage 2 are subject to this uncertainty. At the beginning of stage 3 , the uncertainty in the final disposal costs is narrowed (shown at the bifurcation point between stages 2 and 3 in 2050) through information made available, which may come in the form of engineering studies, repository construction data, etc. Decisions made during stage 3 utilize this new information that acts to filter the possible outcomes for the final disposal costs. For example, if this preliminary information indicated that the final disposal cost was higher than the original medium expected value, it is assumed that the probability of the low or medium-low end-states occurring is zero (dashed lines from the medium-high expected cost in Fig. 3.2 only reach the medium, medium-high, and high end-states). At the 2070 resolution date (beginning of stage 4), the repository opens and final disposal costs are fixed until the foreseeable future, represented by solid lines in Fig. 3.2. Then, decisions in stage 4 are made with this information, and agents may choose to alter their fuel cycle policy accordingly.

\subsection{VEGAS Nuclear Fuel CyCle Simulator}

VEGAS, a dynamic simulation tool for the nuclear economy, was reviewed in (Juchau et al., 2010) ${ }^{5}$. It is encoded in Java, and a MATLAB script is available for use in generating output data plots. The alpha version was originally developed as a lightweight, fast-executing platform for scoping nuclear fuel cycle scenarios in order to select a few for more detailed analysis using a simulator such as NFCSim (Schneider et al., 2005). Likewise, the VEGAS code is chosen here as the analysis platform as it provides a physical

\footnotetext{
${ }^{5}$ Contact: eschneider@mail.utexas.edu for questions and comments concerning the VEGAS nuclear fuel cycle simulator.
} 
and economic model of the nuclear fuel cycle while offering a reduced runtime over more detailed fuel cycle systems tools. A reduced runtime is achieved by foregoing distinctions between reactors and fuel cycle facilities of the same general type, tracking material as continuously flowing streams rather than discrete batches, consolidating material flows into essential components (U, Pu, minor actinide (MA), and FP mass flows), and omitting radioactive decay.

Available to each VEGAS simulation are customizable ${ }^{6}$ reactor types, as well as a legacy stockpile of $\mathrm{SNF}^{7}$. Reactor types are subcategorized by recycling tier: tier 0 reactors use virgin feed only, tier 1 reactors operate on a thermal-neutron spectrum and at least some feed comes from tier 0 or 1 fuel discharges, and tier 2 reactors operate on a fast-neutron spectrum with at least some feed coming from tier 0 or 1 fuel discharges. Five front end (U mining and milling, conversion to $\mathrm{UF}_{6}$, enrichment, fuel fabrication, and transportation to reactor site) and seven back end (SNF storage, transportation, and disposal; UF reprocessing; and HLW vitrification, storage, and disposal) fuel cycle technologies are included in each simulation. However, the number and type of front and back end fuel cycle technologies is also fully customizable ${ }^{8}$. The VEGAS simulator deploys generating capacity based on a user-defined electricity generation profile using the inputs listed in Table 3.1. New reactors are deployed in the VEGAS simulation to handle retirement of old

\footnotetext{
${ }^{6} \mathrm{~A}$ mass flow connecting the input front and back end fuel cycle technologies must be defined for each input reactor type.

7 The legacy stockpile of SNF is set to 0 for the scenarios in this work. A technical review by Oak Ridge National Laboratory found that approximately 98 percent of the total current inventory of commercial SNF may be disposed without the option for retrievability for reuse or research purposes (Wagner et al., 2013). The review is meant to support a comprehensive national fuel cycle strategy, and notes that disposal of the legacy stockpile does not presuppose that it has no value.

${ }^{8}$ See 6.
} 
reactors and demand changes, according to the input "Try To Build" scenario (i.e. try to build 75 percent LWRs and 25 percent FRs starting in year $2050^{9}$ ).

Table 3.1. Electricity generation profile input data Item

Unit (data type)

\begin{tabular}{|c|c|}
\hline \multicolumn{2}{|l|}{ A. Initial reactor fleet data } \\
\hline Initial Generation Capacity & $\mathrm{GW}_{\mathrm{e}}$ (integer) \\
\hline Year Initial Fleet Begins Retiring ${ }^{\mathrm{a}}$ & yr (integer) \\
\hline Year Initial Fleet Finishes Retiring ${ }^{a}$ & yr (integer) \\
\hline \multicolumn{2}{|l|}{ B. Initial generating capacity } \\
\hline Capacity Data ${ }^{b}$ & reactor number (integer), \% (float) \\
\hline \multicolumn{2}{|c|}{ C. Exogenous demand for nuclear energy } \\
\hline Target Generation Capacity ${ }^{\mathrm{c}}$ & $\mathrm{GW}_{\mathrm{e}}$ (integer) \\
\hline Growth Rate $^{\mathrm{d}}$ & $\% / y r$ (float) \\
\hline \multicolumn{2}{|l|}{ D. Reactor "Try To Build" scenario } \\
\hline Try To Builde & yr (integer); reactor number (integer), \% (float) \\
\hline \multicolumn{2}{|c|}{$\begin{array}{l}\text { a Initial fleet retires at a constant rate during this interval } \\
\text { b Sum of percentages for initial capacity data must add to } 100 \text {; any number of reactor-percentage pairs may be } \\
\text { c Linear growth from previous year is assumed to reach target generating capacity at specified year } \\
\text { d Demand growth rate applied to this and subsequent years } \\
\text { e } \\
{ }^{\text {Toly }} \text { Try to deploy this breakdown of reactor generating capacity; any number of reactor-percentage pairs may be } \\
\text { specified. Will default to lower-tier facility is feed unavailable. }\end{array}$} \\
\hline
\end{tabular}

The annual $\mathrm{U}, \mathrm{Pu}$, and $\mathrm{MA}$ requirements for fresh reactor fuel in units of $\mathrm{kg}(\mathrm{U}, \mathrm{Pu}$, or MA) per $\mathrm{kW}_{\mathrm{e}}$ is derived using Eq. 3.1 and reactor inputs from Table 3.2A. Note, the demand for the three different fuel components is determined by applying Eq. 3.1 with the corresponding value for $x$, shown in Table 3.2B.

$$
\text { Annual Fuel Requirement }=\frac{x \cdot c}{\beta \cdot(1-r) \cdot \eta \cdot 10^{3}} \quad \text { Eq. } 3.1
$$

\footnotetext{
${ }^{9}$ In this scenario, VEGAS would build 3 LWRs, then a FR (assuming their generating capacities are equal), then 3 more LWRs, before another FR. Otherwise, if the demand increased each year by the generating capacity of a single reactor, only LWRs would be built. Using this list for reactor orders, VEGAS would build a FR in the $4^{\text {th }}$ year.
} 
Given the demand for reactor fuel components, the VEGAS code ensures that fuel requirements are fulfilled each year using the algorithm depicted in Fig. 3.3 and is described in the following text. The highest tiered reactor's fuel demand is processed first according to its reprocessing hierarchy. The reprocessing hierarchy associated with each reactor determines the order that UF is reprocessed from other reactors in order to obtain fresh fuel for that reactor. For example, if the reprocessing hierarchy for Reactor 1 is " 2,3 , 4", UF from Reactor 2 is reprocessed first to obtain fuel required by Reactor 1, then Reactor 3, and then Reactor 4. The amount of $\mathrm{U}, \mathrm{Pu}$, and MA from reprocessing is determined by a reprocessing efficiency and output fuel recipe (see Table 3.2B). The reprocessing efficiency is determined by Eq. 3.2 using a user-input percent of feed loss value, $p$. If enriched $\mathrm{U}$ is required for a reactor, the amount of $\mathrm{NU}$ and $\mathrm{SWU}{ }^{1}$ required to achieve that demand is calculated using a ${ }^{235} \mathrm{U}$ tails assay defined by the user.

$$
\text { Reprocessing Recovery Fraction }=1-\frac{p}{100} \quad \text { Eq. } 3.2
$$

Table 3.2. Selected reactor inputs for VEGAS

A. Reactor power variables

\begin{tabular}{|c|c|c|c|}
\hline \multirow[b]{2}{*}{ Plant Size $(s)$} & \multicolumn{3}{|l|}{ Unit (data type) } \\
\hline & \multicolumn{3}{|l|}{$\mathrm{MW}_{\mathrm{e}}$ (integer) } \\
\hline Thermal Efficiency $(\eta)$ & \multicolumn{3}{|l|}{$\frac{\mathrm{MW}_{e}}{\mathrm{MW}_{t}}$ (float) } \\
\hline Recirculating Power $(r)$ & \multicolumn{3}{|c|}{$\frac{\mathrm{MW}_{\mathrm{e}} \text { recycled to plant systems }}{\mathrm{MW}_{\mathrm{e}} \text { produced }}$ (float) } \\
\hline Discharge Burnup $(\beta)$ & \multicolumn{3}{|c|}{$\frac{\mathrm{MWd}_{t}}{\mathrm{~kg} \mathrm{IHM}}$ (integer) } \\
\hline Availability $(c)$ & \multicolumn{3}{|c|}{ Load factor (float) } \\
\hline \multicolumn{4}{|l|}{ B. Reactor fuel recipe } \\
\hline & $\begin{array}{c}\mathrm{U} \\
{[\mathrm{kg} / \mathrm{kg} \mathrm{IHM}]} \\
\end{array}$ & $\begin{array}{c}\mathrm{Pu} \\
{[\mathrm{kg} / \mathrm{kg} \mathrm{IHM}]}\end{array}$ & $\begin{array}{c}\text { MA } \\
{[\mathrm{kg} / \mathrm{kg} \mathrm{IHM}]} \\
\end{array}$ \\
\hline Input Fractional Composition & $x_{U}$ & $x_{P u}$ & $x_{M A}$ \\
\hline Output Fractional Composition ${ }^{\mathrm{a}}$ & $y_{U}$ & $y_{P u}$ & $y_{M A}$ \\
\hline
\end{tabular}


Fuel requirements may be limiting in either of two cases: (1) the heavy metal yield from reprocessing all UF in a reactor's hierarchy is insufficient to fulfill the demand, or (2) the heavy metal yield from utilizing all reprocessing capacity for that reactor's tier is insufficient to fulfill the demand. If either of these two cases occur, the most recently-built reactor of the type lacking fuel is removed from the electricity generation profile and is replaced with equivalent generating capacity from a reactor(s) of a lower tier. A prioritized list of lower-tier reactors is supplied by the user. The last entry in the list must be a tier 0 reactor that uses $U$ fuel, of which there is an unrestricted supply. This new electricity generation profile is then used to define a new $\mathrm{U}, \mathrm{Pu}$, and MA demand profile, and the algorithm continues until all reactor fuel demands can be met.

\subsubsection{VEGAS LeVElized COST OF EleCtRiCity CALCUlation}

Each VEGAS simulation calculates the annual LCOE in cents per kWh of electricity produced. The LCOE is the price at which electricity must be generated in order to break even over the lifetime of the simulation ${ }^{10}$ and consists of three components: front end fuel cycle charges, back end fuel cycle charges, and reactor charges. Default unit costs are assigned to each distinct fuel cycle process in VEGAS, though unit costs may also be associated with a given reactor technology. For example, FR fuel fabrication may be assigned a larger unit cost than LWR fuel fabrication due to difficulty of fuel handling. Unit costs are given in dollars per unit of mass throughput, typically $\mathrm{kg} \mathrm{U}$, IHM, or SWU. An independent unit cost is defined for separated actinide (TRU) storage and disposal. Reactor cost inputs are broken into capital costs (dollars) and annual

\footnotetext{
${ }^{10}$ Simulations are carried out through an additional lifetime of the longest operating facility to ensure liability costs are accounted for. For example, results from VEGAS simulations in this study are analyzed for the time period [2015, 2100], but an actual simulation is carried through to 2160 , assuming a reactor operating lifetime of 60 years. Otherwise, a FR may be built at the "end" of the time period despite a fuel shortage occurring in the future.
} 
operations and maintenance costs (dollars per year). In addition, an option has been added for calculation of fuel cycle unit costs using a similar method.

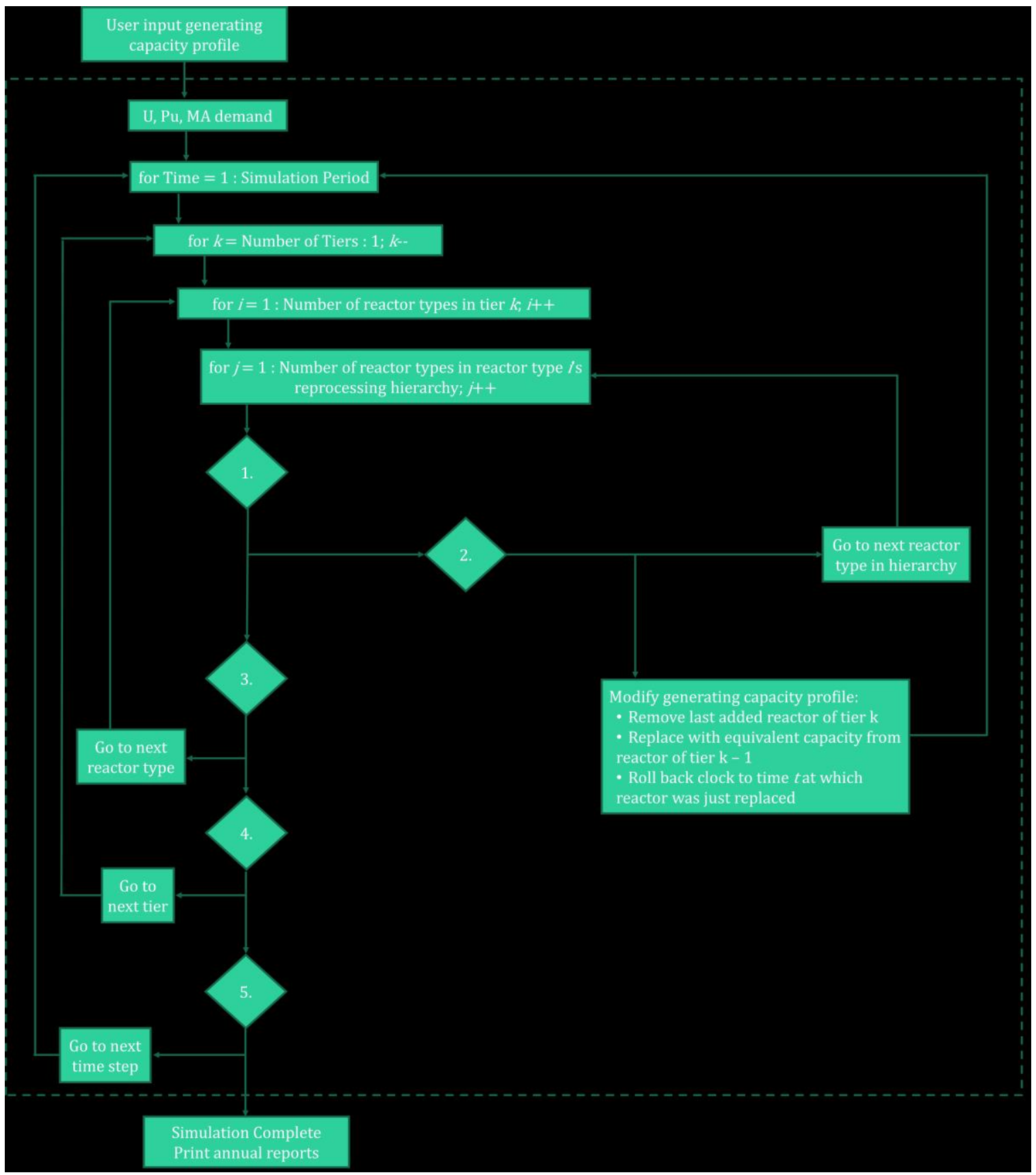

Figure 3.3. Reactor fuel fulfillment algorithm. 
Front-end and back-end mass flows are used to calculate the total fuel cycle cost $\left(C_{F C}\right)$ by Eq. 3.3:

$$
C_{F C}=\sum_{i=1}^{I} M_{i} \cdot U C_{i} \cdot P V F_{i},
$$

where $i=1,2 \ldots I$ indexes fuel cycle technologies, $M_{i}$ is the mass throughput of the $i^{t h}$ technology, $U C_{i}$ is the unit cost of the $i^{t h}$ technology, and $P V F_{i}$ is the present value function (PVF) of the $i^{\text {th }}$ technology. The PVF discounts the charge to the year the technology was employed through lead and lag times input to the VEGAS simulation. Back-end costs are assumed to be covered by a sinking fund from revenues generated while the fuel is in reactors producing electricity. However, the risk-free rate of return is set to zero percent ${ }^{11}$ to avoid results that are driven by discounting-driven benefits associated with deferring a future liability. These back-end costs are not determined until the end of a VEGAS simulation when unit costs are applied to mass flows (part of the Fig. 3.3 "Print annual reports" process).

While default unit costs for fuel cycle technologies are typically employed for Eq. 3.3, an option for dynamic calculation of fuel cycle technology unit costs from capital and operating costs, adapted from the Generation-IV Economic Modeling Working Group (EMWG, 2007), has been added. This dynamic unit cost calculation is employed for LWR UF reprocessing ${ }^{12}$; Table 3.3 lists the data and parameters for this calculation. The option for dynamic calculation of unit costs may be chosen by specifying four parameters for a given fuel cycle technology: (1) the plant size $\left(M_{c}\right),(2)$ the annual capital cost repayment $(A C C)$, (3) the fixed operations and maintenance (FOM) charge, and (4) the variable

\footnotetext{
${ }^{11}$ See (Arrow et al., 1996) discussion on the prescriptive approach to determining the appropriate discount rate for long-range planning.

12 This feature is added only to tier 0 reprocessing capacity. Tier 2 reprocessing capacity (for FRs) is assumed wholly adequate corresponding to builds of co-located reprocessing and fuel fabrication facilities. This technology has previously been demonstrated at the Idaho Integral Fast Reactor facility adjacent to EBR-II (Shropshire et al., 2009).
} 
operations and maintenance $(V O M)$ charge. In addition, a capacity profile for the technology in throughput per year for each year in the simulation must be defined. The $A C C$ is calculated using Eq. 3.4:

$$
A C C=(T O C+I D C) \times A F,
$$

where the interest during construction $(I D C)$ is given in Eq. 3.5,

$$
I D C=\sum_{i=0}^{T_{c}-1} T O C \times f_{i}\left[(1+r)^{T_{c}-i}-1\right],
$$

and the amortization factor $(A F)$ is given in Eq. 3.6,

$$
A F=\frac{r}{1-(1+r)^{-T_{o}}} .
$$

Finally, the unit cost is calculated using Eq. 3.7,

$$
U C=\frac{A C C+F O M}{M}+V O M
$$

According to Eq. 3.7, the unit cost is minimized if the facility is operating at its maximum capacity $\left(M_{c}=M\right)$. Otherwise, the unit cost increases as the facility's actual throughput drops below the facility's maximum capacity. In this way, a penalty is imposed for capacity underutilization, ensuring that reprocessing capacity expansion profiles with excessive idle capacity are not falsely identified as cost-effective. As $A C C$ and $F O M$ are defined for a single facility, specifying the maximum throughput of a fuel cycle facility allows for correct calculation of unit costs for multiple facility capacities.

The second component of the LCOE consists of reactor costs $\left(C_{\text {reactor }}\right)$ that are simply the sum of their respective $A C C$ and FOM charges, Eq. 3.8:

$$
C_{\text {reactor }}=A C C+F O M \text {. }
$$


For reactor costs, VEGAS calculates the ACC from user-inputs in Table $3.3 T O C, T_{o}, T_{c}$, and $r$. VOM costs consist of reactor fuel costs, which are omitted in $C_{\text {reactor }}$ as they are accounted for in $C_{F C}$.

Given $C_{F C}$ and $C_{\text {reactor }}$ specified in cents, the annual LCOE is then given by Eq. 3.9:

$$
L C O E=\frac{C_{F C}+C_{\text {reactor }}}{E},
$$

where $E$ is the total amount of electricity produced in that year in $\mathrm{kWh}$.

Table 3.3. Unit cost calculation parameters

\begin{tabular}{|c|c|c|}
\hline Symbol & Unit & Definition \\
\hline TOC & $\$$ & Total overnight cost \\
\hline FOM & $\$ / y r$ & Fixed operations and maintenance cost \\
\hline$V O M$ & $\$ / \mathrm{kg}$ & Variable operations and maintenance cost \\
\hline$T_{c}$ & $\mathrm{yr}$ & Duration (years) of construction \\
\hline$T_{o}$ & $\mathrm{yr}$ & Duration (years) of operation \\
\hline$M$ & $\mathrm{~kg} / \mathrm{yr}$ & $\begin{array}{l}\text { Annual production (throughput) of product in } \mathrm{kg} \text { of basis unit per yr: } \\
\text { technology-specific basis unit may be } \mathrm{U}, \mathrm{IHM} \text {, SWU, etc. }\end{array}$ \\
\hline$M_{c}$ & $\mathrm{~kg} / \mathrm{yr}$ & Maximum production (throughput) of product in $\mathrm{kg}$ of basis unit per $\mathrm{yr}$ \\
\hline$f_{i}$ & - & $\begin{array}{l}\text { Fraction of TOC expended in year } i \text { of construction (summing } f_{i} \text { over } \\
\text { all years } i=1,2 \ldots T_{c} \text { equals } 1.0 \text { ) }\end{array}$ \\
\hline$r$ & $1 / \mathrm{yr}$ & Real discount rate \\
\hline$A F$ & $1 / \mathrm{yr}$ & (calculated) Capital cost amortization factor \\
\hline$I D C$ & $\$$ & (calculated) Interest during construction \\
\hline$A C C$ & $\$ / y r$ & (calculated) Amortized annual capital cost \\
\hline$U C$ & $\$ / \mathrm{kg}$ & (calculated) Unit cost of product \\
\hline
\end{tabular}

\subsection{Approach to the No-Data Problem}

The VegAS nuclear fuel cycle simulator tool, described in Section 3.2, is used to obtain the LCOE for the various fuel cycle transition strategies. Given the scenario described in Section 3.1, the objectives are two-fold: to identify optimal strategies under 
conditions of perfect information and to identify optimal hedging strategies under the limits of imperfect information.

A description of the suite of VEGAS simulations performed for the fuel cycle transition analysis performed in this work is given in the first subsection. Then, the procedure for identifying strategies under perfect and imperfect information conditions from the VEGAS simulations is described.

\subsubsection{DESCRIPTION OF VEGAS SIMULATIONS}

Full documentation of input parameters to the VEGAS simulator used in the transition scenario examined here is provided in Appendix A. An overview of the scenario is given in Section 3.1.

Each of the five states of nature that may occur (the five end-states depicted in Fig. 3.2) is simulated in VegAS, creating five families of simulations, $S_{i}$ with $i=1,2 \ldots 5$. Table 3.4 gives the cost data for each end-state, along with their associated probabilities. The low, medium-low, and medium disposal cost outcomes represent engineering estimates and are taken from the low, nominal, and high estimates from the Advanced Fuel Cycle Cost Basis report (Shropshire et al., 2009). The medium-high and high disposal cost outcomes represent unforeseen issues with building and operating a geologic repository. The highest disposal cost outcomes are taken as 3 and 10 times the nominal disposal costs given in (Shropshire et al., 2009). The ratio between disposal costs of SNF and vitrified, FP-loaded HLW is taken as the median ratio of those presented in (Shropshire et al., 2009). The median ratio between disposal costs of HLW to SNF is approximately 8 and is applied with costs for SNF as a baseline.

For each possible end-state, a subset of simulations is performed for each reprocessing capacity expansion strategy. The individual strategies arise as follows: at each 10 year increment beginning in 2030 and ending with the simulation in 2100, a 
$1000 \mathrm{tIHM} / \mathrm{yr}$ LWR UF reprocessing facility may be built or not built (see Fig. 3.4). Thus, there are 128 possible reprocessing capacity expansion profiles, each of which are simulated for each end-state $i$. Finally, the entire set of simulations, $S$, is indexed over the 5 end-states by $i$ and 128 expansion profiles by $j$, giving a total of 640 simulations, $S_{i j}$, with $i=1,2 \ldots 5$ and $j=1,2 \ldots 128$.

\section{Table 3.4. SNF and HLW Disposal Costs}

\begin{tabular}{r|ccc} 
& \multicolumn{3}{|c}{ Disposal Costs } \\
End-states & $\begin{array}{c}\text { SNF } \\
\text { (\$/kg IHM) }\end{array}$ & $\begin{array}{c}\text { HLW } \\
\text { (\$/kg IHM in HLW) }\end{array}$ & Probability $^{\mathbf{~} \mathbf{k}}$ \\
\hline Low & 400 & 3,200 & $1 / 9$ \\
Medium-Low & 650 & 5,200 & $2 / 9$ \\
Medium & 1,000 & 8,000 & $1 / 3$ \\
Medium-High & 1,950 & 15,600 & $2 / 9$ \\
High & 6,500 & 52,000 & $1 / 9$
\end{tabular}

In Fig. 3.4, information is made known at times 2050 and 2070 (the addition of " $\delta$ " symbolizes that the information made available that year cannot affect the build/no-build decision since construction must begin prior to that year). However, information made known in 2050 affects decisions made later in 2060 and 2070. Likewise, information learned in 2070 affects decisions made in 2080 and 2090. This may be seen as a ROA approach, as agents may adopt a "wait-and-see" strategy until the uncertainty in disposal cost outcomes is lessened or resolved. Fig. 3.4 may be supplemented by Fig. 3.2, where the chance nodes of Fig. 3.4 (yellow circles) correspond to bifurcation points between stages in Fig. 3.2.

13 The probabilities for each disposal cost end-state occur through the filtration of possible outcomes depicted in Fig. 3.2. In year 2050, information indicates whether disposal costs are expected to be low, median or high, each with a probability of $1 / 3$. This estimate affects the possible end-states moving forward in time. For example, if given a high estimate, end-states 3, 4, and 5 are accessible, each with a probability of $1 / 3$. Applying these probabilities to the branches in Fig. 3. 2 gives the values shown in Table 3.4. 


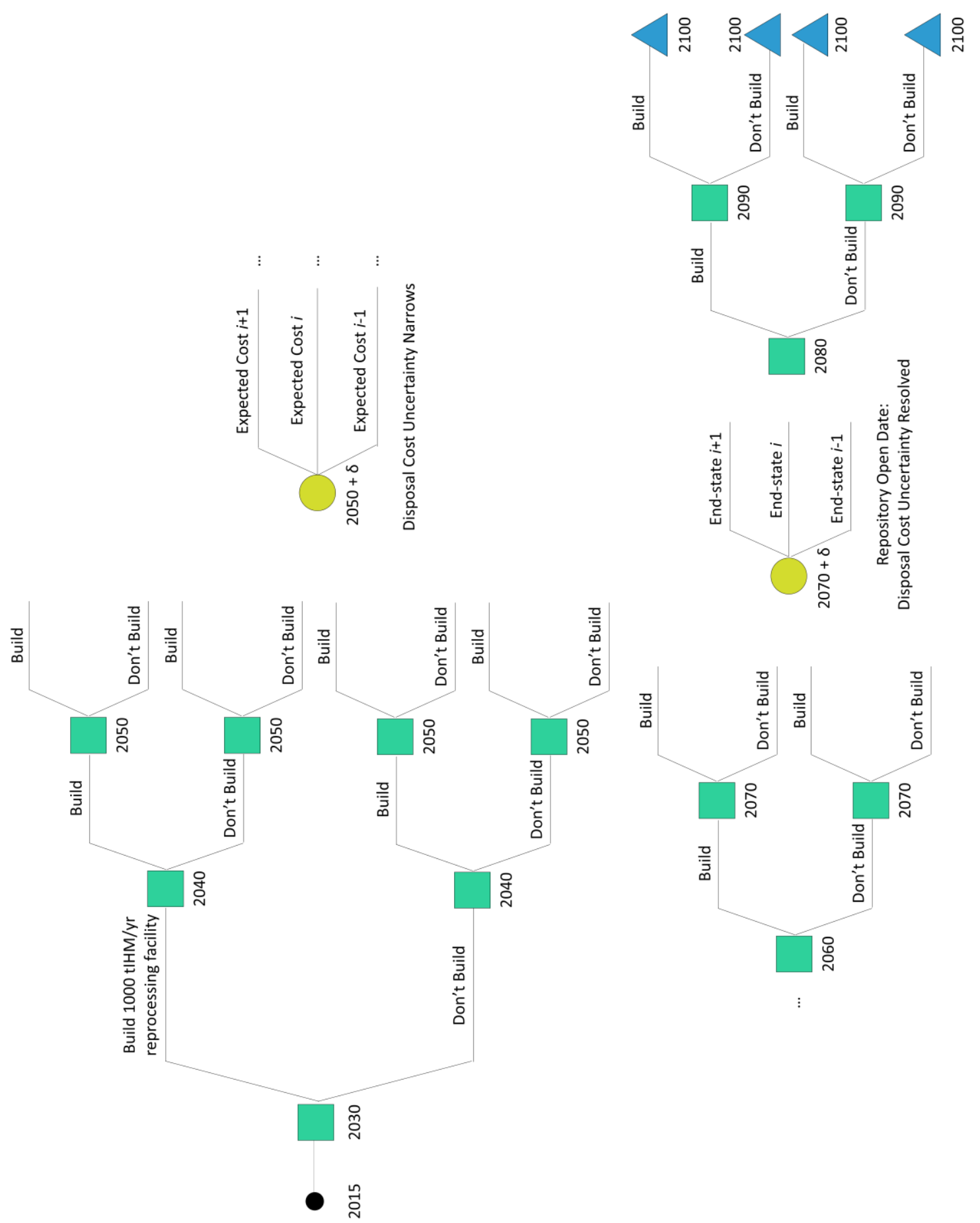

Fig. 3.4. Reprocessing capacity expansion strategies 


\subsubsection{Transition Strategies Under Perfect Information}

For each of the five states of nature that may occur (corresponding to the five endstates listed in Table 3.4), three transition strategies corresponding to distinct objective functions will be identified as optimal. The three objective functions aim to minimize the: (1) average LCOE, (2) maximum LCOE, and (3) energy-integrated LCOE. Let $c_{k}^{i, j}$ be the calculated LCOE for the $i^{\text {th }}$ end-state, the $j^{\text {th }}$ expansion profile and the $k^{\text {th }}$ year, $k=1$, $2 \ldots N^{14}$. Then, for the five possible final disposal costs, the three optimal transition strategies under perfect information are defined as follows:

- Lowest average LCOE

$$
j_{1, i}^{*}=\underset{j}{\operatorname{argmin}} \frac{1}{N} \sum_{k=1}^{N} c_{k}^{i, j}
$$

- Lowest maximum LCOE

$$
j_{2, i}^{*}=\underset{j}{\operatorname{argmin}}\left\{\max _{k} c_{k}^{i, j}\right\}
$$

- Lowest integrated LCOE

$$
j_{3, i}^{*}=\underset{j}{\operatorname{argmin}} \sum_{k=1}^{N} c_{k}^{i, j} E_{k},
$$

where $E_{k}$ is the electricity produced in year $k$. The strategy $j$ that is found as optimal is then denoted $j_{\alpha, i}^{*}$ for the $\alpha$ objective function $(\alpha=1,2,3)$ and $i^{\text {th }}$ end-state.

Each objective function is applied to the $S_{i j}$ suite of simulations, obtaining $15 j^{*}$ perfect information strategies (see Fig. 3.5). These strategies represent those which perform the best for each final disposal cost outcome and objective function.

\footnotetext{
14 The value of $N$ is 86 , corresponding to 86 years from 2015 to 2100 in a simulation.
} 


\subsubsection{HEDGING STRATEGIES UNDER IMPERFECT INFORMATION}

Given the $j_{\alpha, i}^{*}$ optimal transition strategies found under perfect information, it is then possible to identify optimal hedging strategies under imperfect information. The regret $(r)$ for following a different strategy other than the perfect information strategy represents foregone savings. The regret is calculated differently for each objective function; for the lowest average and maximum LCOE objective functions, $r$ has units of cents per kWh of electricity produced, whereas for the lowest integrated LCOE objective function, $r$ has units of dollars. The regrets are defined as follows:

- lowest average LCOE

$$
r_{1}=\frac{1}{N}\left(\sum_{k=1}^{N} c_{k}^{j, i}-c_{k}^{j_{1, i}^{*}}\right)
$$

- lowest maximum LCOE

$$
r_{2}=\max _{k} c_{k}^{i, j}-\max _{k} c_{k}^{j_{2, i}^{*}}
$$

- lowest integrated LCOE

$$
r_{3}=\sum_{k=1}^{N} c_{k}^{i, j} E_{k}-c_{k}^{j_{3, i}^{*}} E_{k}
$$

Two choice criteria $g_{\beta}$ with $\beta=1,2$ are used to select the optimal hedging strategies: (1) the minimax regret, and (2) the expected regret. The first choice criterion is independent of the probability distribution on the cost outcomes, whereas the second criterion is heavily dependent on the probability distribution of the cost outcomes. The two choice criteria are defined as follows: 
- Minimax regret

$$
g_{1, \alpha}^{*}=\underset{j}{\operatorname{argmin}}\left\{\max _{i} r_{\alpha}\right\}
$$

- Lowest expected regret

$$
g_{2, \alpha}^{*}=\underset{j}{\operatorname{argmin}} \sum_{i=1}^{I} P_{i} r_{\alpha}
$$

where the notation $g_{\beta, \alpha}^{*}$ indicates that the $\beta$ choice criterion is applied to the subset of $j_{\alpha, i}^{*}$ strategies, $P_{i}$ is the probability of the $i^{\text {th }}$ end-state occurring (see Table 3.4), and $I$ is the total number of end-states, 5. Six hedging strategies will be identified for stage 215 of the simulation (Fig. 3.2), corresponding to the three objective functions $j_{\alpha}$ and the two choice criteria $g_{\beta}$ (see Fig. 3.5). These hedging strategies account for the uncertainty in which disposal cost will be realized in 2070 , the repository opening date.

At the beginning of stage 3 , more information is made available about the final cost of disposal in the repository, which allows for a filtration of the possible outcomes. Therefore, eighteen total hedging strategies are found for stage 3, corresponding to the three objective functions $j_{\alpha}$, the two choice criteria $g_{\beta}$, and the three filtrations. The procedure for identifying the hedging strategies in stage 3 is not explicitly defined here. Simply put, a new set of $j_{\alpha, i}^{*}$ perfect information strategies is identified, but with the strategies $S_{j}$ restricted to those that follow the hedging strategy employed in stages 1 and 2 until the 2050 date. Then the hedging strategies in stage 3 are identified among the $S_{j}$ subset of strategies using the new set of $j_{\alpha, i}^{*}$ perfect information strategies. Following the

\footnotetext{
15 There are no decisions made in stage 1 as only the once-through LWR fuel cycle may be followed. Therefore, stage 1 has no bearing on the hedging strategy identified.
} 
resolution date (repository open date), a unique strategy is followed in stage 4 for each possible final SNF and HLW disposal cost.

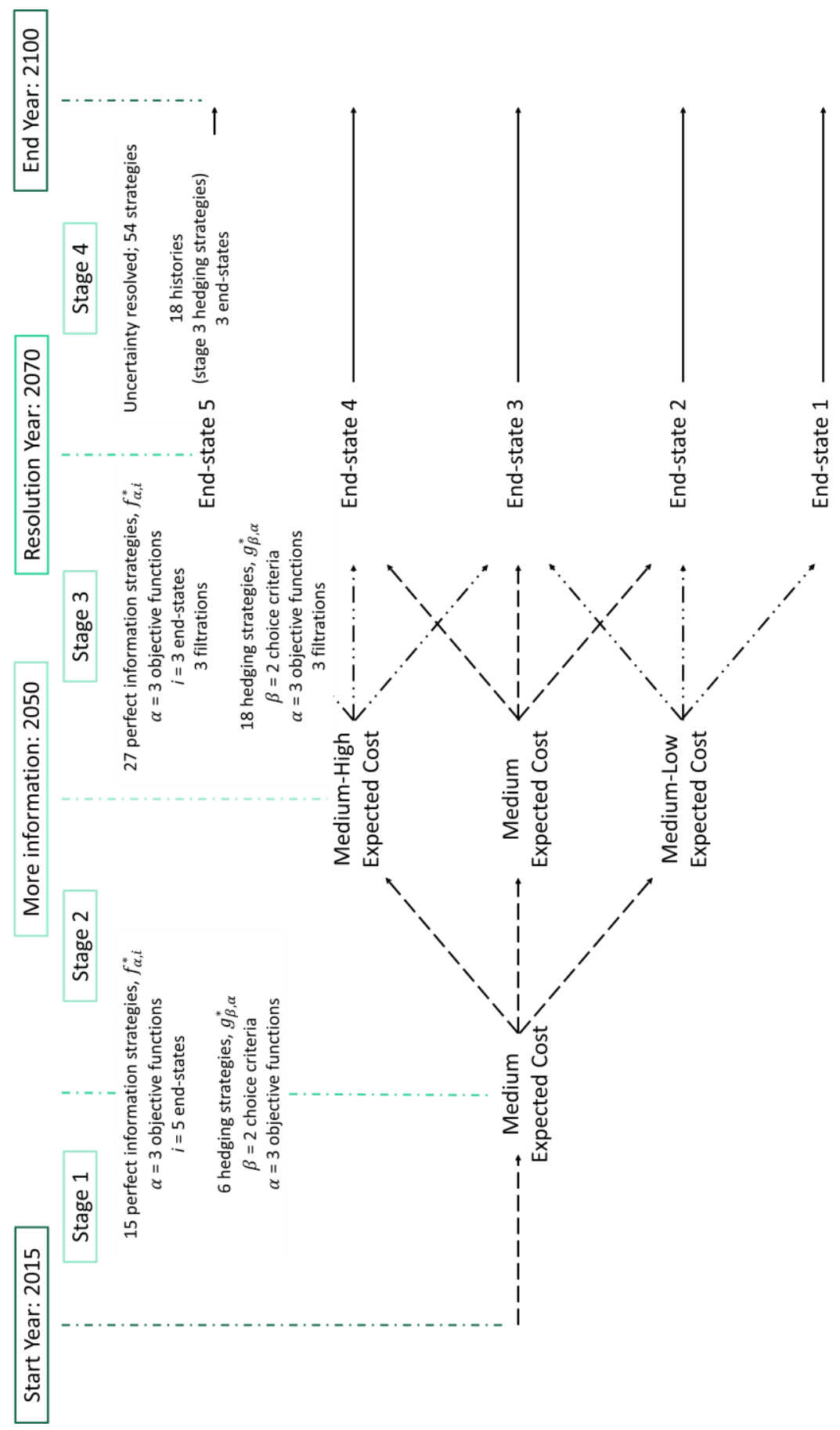

Fig. 3.5. Perfect information and hedging strategies during simulation time frame. 


\section{Chapter 4: Results}

This chapter presents results from the reference transition scenario discussed in Chapter 3. In addition, two sensitivity studies were performed which focus on varying the ratio between HLW and SNF disposal costs and FR capital costs. The methodology reviewed in Chapter 3 could be extended to include these, as well as other, uncertainties as part of the decision framework, and may be the subject of future work.

Each scenario is defined by input data for the VEGAS fuel cycle simulator found in Appendix A. The data includes electricity generation profiles, reactor power parameters and fuel input/output recipes, fuel cycle unit costs, and reactor capital costs. The primary data relevant for each scenario is presented along with the corresponding subsection.

\subsection{REFERENCE SCENARIO}

A summary table of the reference transition scenario input parameters is given in Table 4.1. A full description of the reference scenario is given in Chapter 3 with VEGAS input parameters available in Appendix A. This scenario considers a transition from the current once-through LWR fuel cycle to one in which separated TRU from LWR UF is continuously recycled in FRs. The VEGAS simulation begins in 2015 with the current $100 \mathrm{GW}_{\mathrm{e}} \mathrm{LWR}$ fleet, a nuclear electricity demand growth rate of 2 percent per year, and a geologic waste repository slated to open in 2070. The VEGAS simulations are carried out through 2160 (an additional lifetime of the longest operating facility) to ensure liability costs are accounted for. However, only the 2015 to 2100 period is used for the decision analysis.

At each 10 year increment beginning in 2030 and ending in 2100, a 1,000 tIHM/yr LWR UF reprocessing facility may be built or not built; a total of 7 units may be added this way. Since VeGas builds FRs at the maximum rate that the existing reprocessing 
capacity supports, a simulation is fully specified by its reprocessing capacity expansion profile.

Table 4.1. Summary of reference scenario input parameters.

\begin{tabular}{|c|c|c|c|c|}
\hline Year & Item & Value & Unit & Reactor Type \\
\hline \multicolumn{5}{|c|}{ A. Simulation Parameters } \\
\hline 2015 & Start Year & 2015 & $\mathrm{yr}$ & - \\
\hline 2100 & Decision-making End Year & 2100 & $\mathrm{yr}$ & - \\
\hline 2160 & Simulation End Year & 2160 & $\mathrm{yr}$ & - \\
\hline 2015 & Legacy SNF Stockpile & 0.0 & tIHM & - \\
\hline 2015 & Electricity Demand Growth Rate & 2.0 & $\% / y r$ & - \\
\hline 2070 & Repository Open Date & 2070 & $\mathrm{yr}$ & - \\
\hline \multicolumn{5}{|c|}{ B. Reactor Fleet Data } \\
\hline 2015 & Initial Generation Capacity & 100 & $\mathrm{GW}_{\mathrm{e}}$ & - \\
\hline 2015 & Initial Capacity Data & 100 & $\%$ & PWR \\
\hline 2020 & Year Initial Fleet Begins Retiring & 2020 & $\mathrm{yr}$ & - \\
\hline 2050 & Year Initial Fleet Finishes Retiring & 2045 & $\mathrm{yr}$ & - \\
\hline- & New Reactor Lifetime & 60 & $\mathrm{yr}$ & - \\
\hline- & Reactor Construction Time & 4 & $\mathrm{yr}$ & - \\
\hline \multicolumn{5}{|c|}{ C. Reactor "Try To Build" scenario } \\
\hline 2015 & Try To Build & 100 & $\%$ & PWR \\
\hline 2020 & Try To Build & 100 & $\%$ & APWR \\
\hline \multirow{2}{*}{2030} & \multirow{2}{*}{ Try To Build } & 50 & $\%$ & APWR \\
\hline & & 50 & $\%$ & FR \\
\hline
\end{tabular}

The costs of SNF and HLW disposal in the repository are uncertain, with the five possible end-state cost outcomes and their respective probabilities given in Table 4.2. For the reference scenario, the ratio between HLW and SNF disposal costs is 8 , the approximate median ratio given in (Shropshire et al., 2009). The VEGAS simulator delays calculating the annual LCOE until the end of the simulation period, when it uses mass flows to determine if fuel was disposed or reprocessed. Back end costs are ascribed to the year that fuel is discharged, and are calculated using a zero percent discount rate. 
Table 4.2. SNF and HLW Disposal Costs

\begin{tabular}{r|ccc} 
End-states & $\begin{array}{c}\text { SNF } \\
\text { (\$/kg IHM) }\end{array}$ & $\begin{array}{c}\text { HLW } \\
\text { (\$/kg IHM in HLW) }\end{array}$ & Probability \\
\hline Low & 400 & 3,200 & $1 / 9$ \\
Medium-Low & 650 & 5,200 & $2 / 9$ \\
Medium & 1,000 & 8,000 & $1 / 3$ \\
Medium-High & 1,950 & 15,600 & $2 / 9$ \\
High & 6,500 & 52,000 & $1 / 9$
\end{tabular}

Fig. 4.1 gives the annual LCOE for each reprocessing capacity expansion profile and the medium-low, medium-high and high SNF and HLW disposal cost end-states ${ }^{16}$. The two groupings of data points in Fig. 4.1a-c roughly correspond to disposal and reprocessing of LWR UF. The solid white line extending from 2015 to 2100 in Fig. 4.1a-c coincides with the once-through fuel cycle, in which all UF is directly disposed and no reprocessing facilities are ever built. If a single reprocessing unit is built during the simulation, the LCOE in 2015 is elevated (Fig. 4.1a-b) or drops (Fig. 4.1c), depending on the relative costs of reprocessing and disposal, since the oldest UF is reprocessed first. For cases where up to 3 reprocessing units are built, the installed reprocessing capacity is insufficient to sustain a complete transition to the synergistic LWR-FR fuel cycle that is achieved when a larger number of reprocessing units are built. In these situations, the LCOE will eventually approximate that of the direct disposal strategy as fuel discharged past a certain date is no longer reprocessed. However, since some UF was reprocessed, the LCOE remains elevated (Fig. 4.1a-b) or deflated (Fig. 4.1c) due to existing FR infrastructure.

\footnotetext{
${ }^{16}$ Data for the low and medium end-states do not differ significantly from that of the medium-low end-state, and so were excluded from Fig. 4.1. Similarly, the trends seen and discussed from the medium-low end-state are also observed in the low and medium end-states, and are excluded from the text.
} 

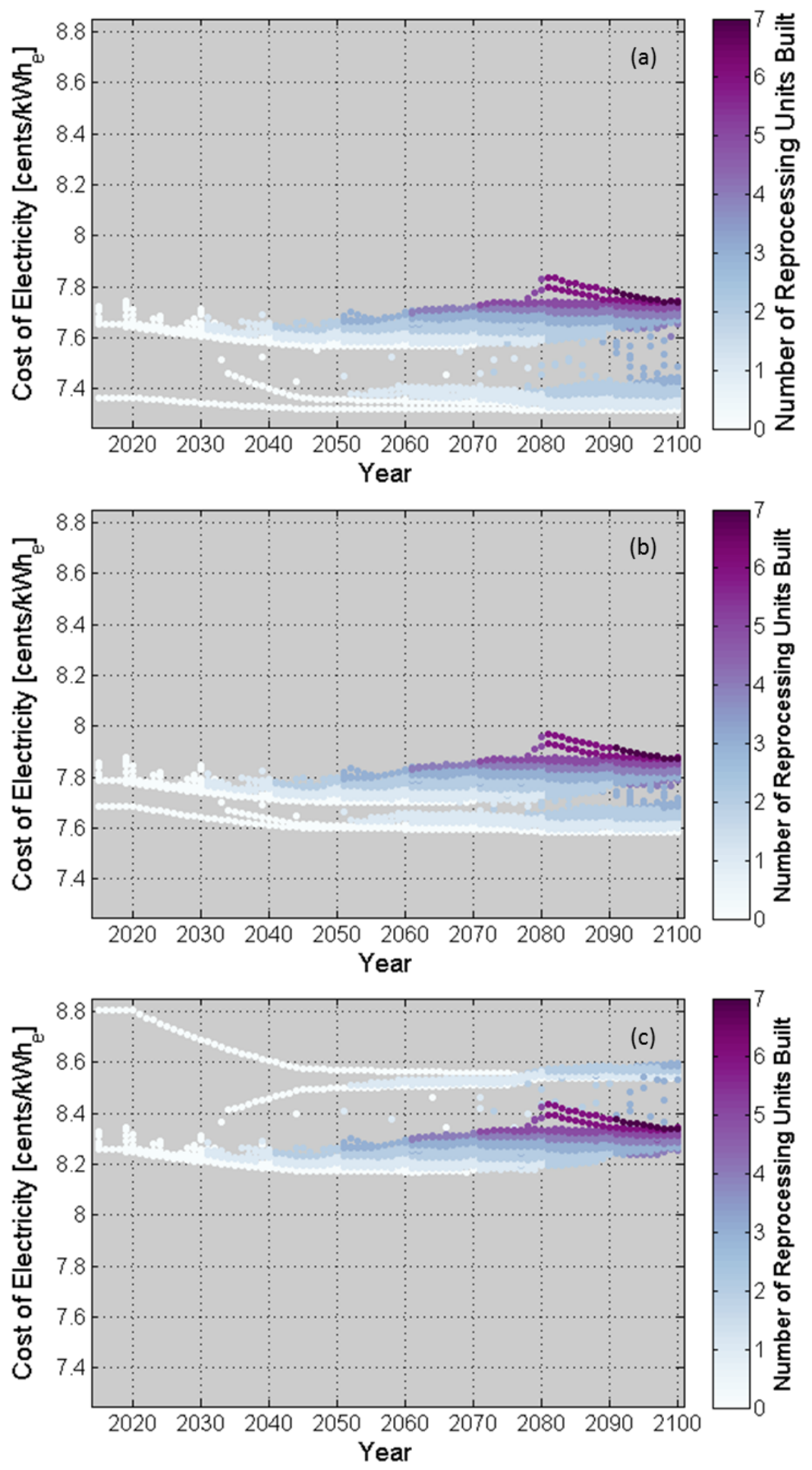

Fig. 4.1. Annual LCOE for all reprocessing capacity expansion profiles and (a) medium-low, (b) medium-high and (c) high SNF and HLW disposal cost outcomes. 
For the medium-low and medium-high disposal cost outcomes (Fig. 4.1a-b), a reprocessing-based fuel cycle is costlier than the direct disposal strategy, whereas for the high disposal cost outcome, the opposite is true (Fig. 4.1c). Relative to Fig. 4.1 a however, when the medium-high disposal cost outcome is realized, the relative different between the reprocessing-based and direct disposal fuel cycles is lessened. For both the medium-high and high outcomes, the LCOEs are elevated due to the higher cost of disposal.

Early in the simulation the LCOE decreases from 2020 to 2045 due to retirement of existing LWRs, which are replaced with advanced LWRs that benefit from increased burnup. This trend is most readily seen in the case where no reprocessing capacity is installed (bottom white line of Fig. 4.1a-b; top white line of Fig. 4.1c) and all LWR fuel is directly disposed. The trend is most dramatic in Fig. 4.1c where the end-state realized is the high disposal cost. Here, the higher disposal cost amplifies the benefit from changeover to more fuel efficient reactors.

The number of years' worth of UF reprocessed is heavily dependent on when UF reprocessing facilities are built. In Fig. 4.1, the earliest the shift in the LCOE is seen is approximately 2030. Here, a single reprocessing unit is built in 2090, allowing for UF generated between 2015 and 2030 to be reprocessed. However, if the unit is built earlier than 2090, UF generated in years following 2030 may be reprocessed, due to a longer operating time of the facility during the simulation.

Fig. 4.2 gives the dynamically calculated reprocessing unit cost for each of the reprocessing capacity expansion profiles, plotted against the year the fuel was reprocessed. For cases where 6 or more reprocessing units are built by 2080, a sharp increase in the LCOE is seen (Fig 4.1) due to elevated reprocessing unit costs (Fig. 4.2). Then, if another reprocessing unit is built in 2090, its capacity is heavily underutilized as the existing units 
suffice to reprocess all LWR UF. This underuse causes the reprocessing unit cost to increase dramatically.

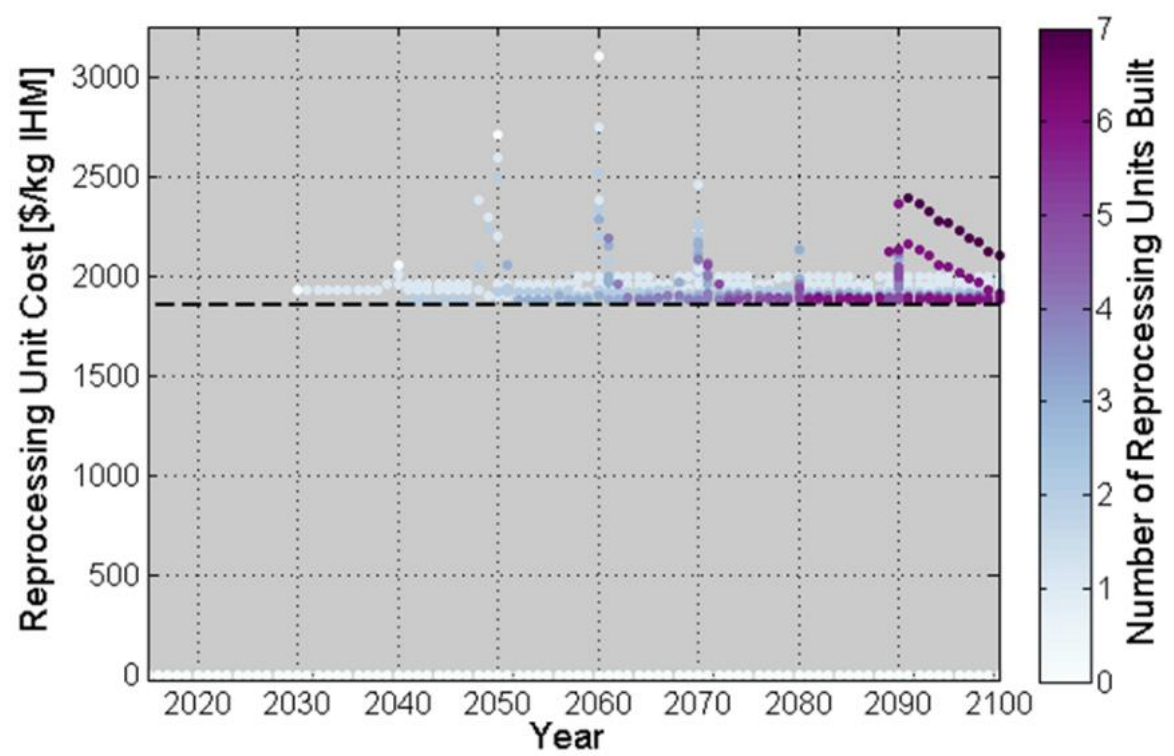

Fig. 4.2. Dynamically calculated reprocessing unit cost for all reprocessing capacity expansions profiles (multi-colored dots) for year fuel reprocessed. Black dashed line represents the frequently employed default unit cost.

For each of the disposal cost outcomes except the high outcome, the perfect information strategy is to directly dispose LWR fuel while refraining from building any reprocessing facilities. Said differently, if agents knew that the disposal cost finally revealed in 2070 would be the low, medium-low, medium or medium-high end-state, they would not move forward with a transition to a reprocessing-based fuel cycle. However, the perfect information strategies for the high end-state entail a transition to the reprocessingbased fuel cycle, see Fig. 4.3. The strategies selected by the 3 objective functions each call for the construction of $41,000 \mathrm{tIHM} / \mathrm{yr}$ reprocessing units. These strategies may not seem to be an aggressive transition to the reprocessing-based fuel cycle, but, as shown in Fig. 4.2 , building up to 6 or 7 reprocessing units may lead to elevated costs due to idle capacity. 


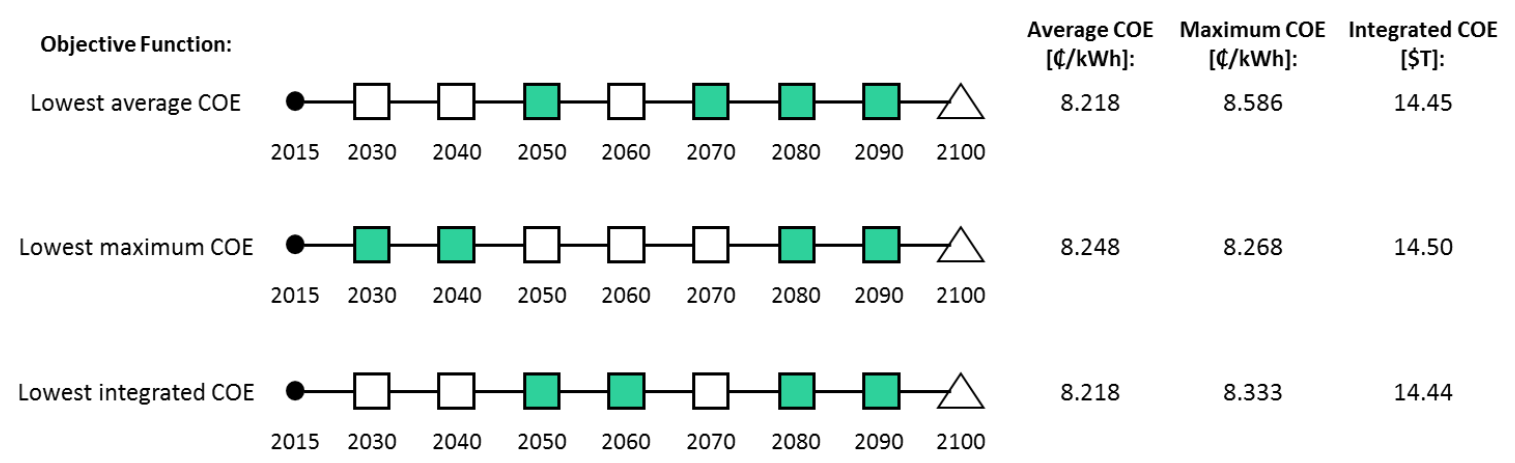

Fig. 4.3. Perfect information strategies for high disposal cost outcome. Green squares indicate construction of a reprocessing unit; white squares indicate foregone build opportunity.

The LCOE for the three perfect information strategies of Fig. 4.3 is shown in Fig. 4.4. The strategy selected by the lowest average LCOE objective function has sufficient reprocessing capacity to reprocess LWR UF discharged prior to 209917. A sharp increase in the LCOE is seen in years 2099 and 2100 as fuel discharged in these years is primarily disposed. Conversely, sufficient reprocessing capacity is installed by the strategy identified by the lowest integrated LCOE objective function. While the only difference between the two strategies is timing of the second reprocessing unit addition, the slight change allows for all fuel to be reprocessed during the decision-making time period when the second unit is built earlier. As a matter of fact, both strategies selected through the lowest average and lowest integrated LCOE objective functions enjoy a lower LCOE through a majority of the decision-making time period. However, due to elevated reprocessing unit costs early in the simulation, and insufficient reprocessing capacity near the end of the decision-making time period (in the case of the lowest average LCOE objective function), neither of these strategies is selected by the lowest maximum LCOE objective function.

17 The optima selected through the various objective functions can be heavily influenced by both the simulation time period and the decision-making time period. In this case, reprocessing units operating past the current simulation time period (2015 to 2160) may eventually reprocess the fuel discharged in 2100 (the end of the decision-making time period). 


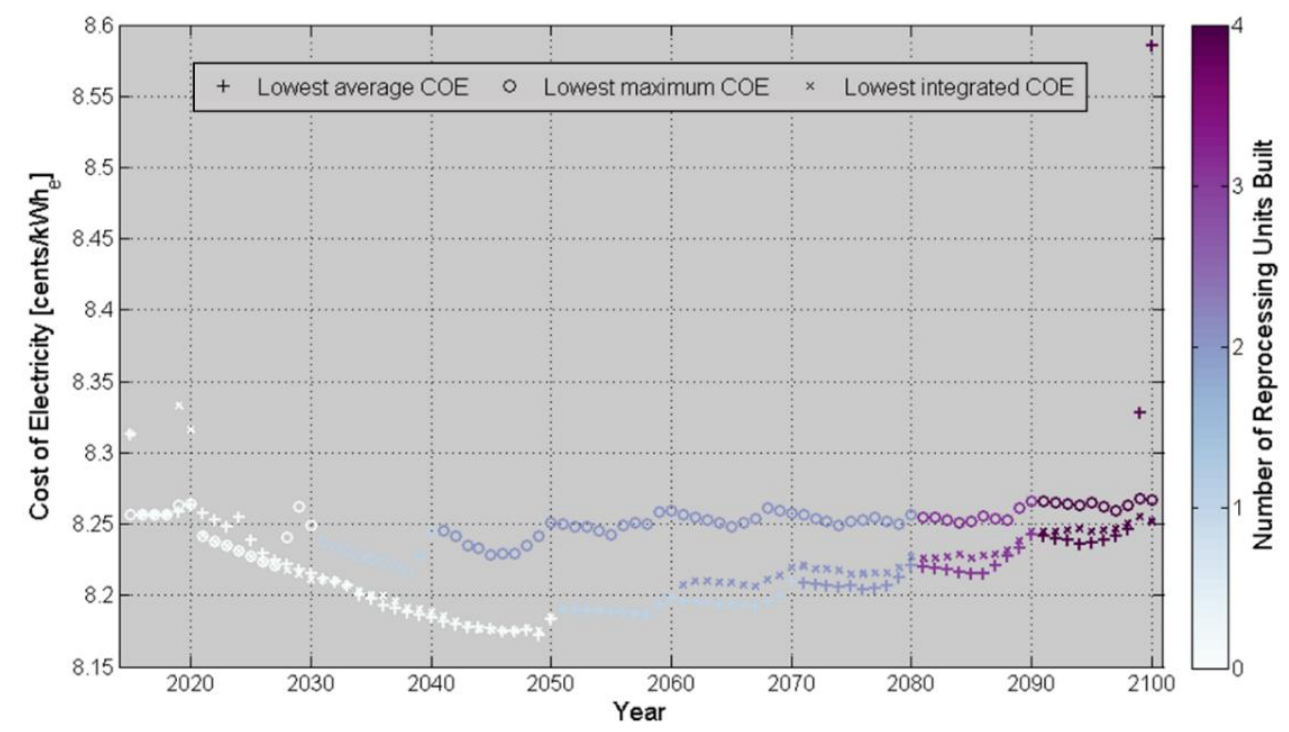

Fig. 4.4. LCOE for perfect information strategies corresponding to high end-state.

The optimal hedging strategies for each objective function and choice criterion pair are shown in Fig. 4.5, along with the regrets accumulated by the hedging strategies selected and the end-states realized. The strategies identified using the minimax regret choice criterion suggest building more reprocessing capacity than in the case of the expected regret choice criterion. This follows as the minimax regret choice criterion is independent of the probability distribution on the possible end-states, and instead acts to minimize the regrets seen in a worst-case scenario. On the other hand, the expected regret choice criterion is heavily dependent on the probability distribution. The hedging strategies identified using this choice criterion reflect that the perfect information strategies for 4 out of the 5 end-states (with a collective $8 / 9$ probability of transpiring) advocate that no transition be made. While the average regret across all end-states is lower for the expected regret choice criterion, when a nonzero regret is incurred for an end-state, it is typically larger than the regret seen using the minimax regret choice criterion. This occurs when the high disposal cost outcome is the end-state realized, and reprocessing units must be built quickly in order to "catch up". 


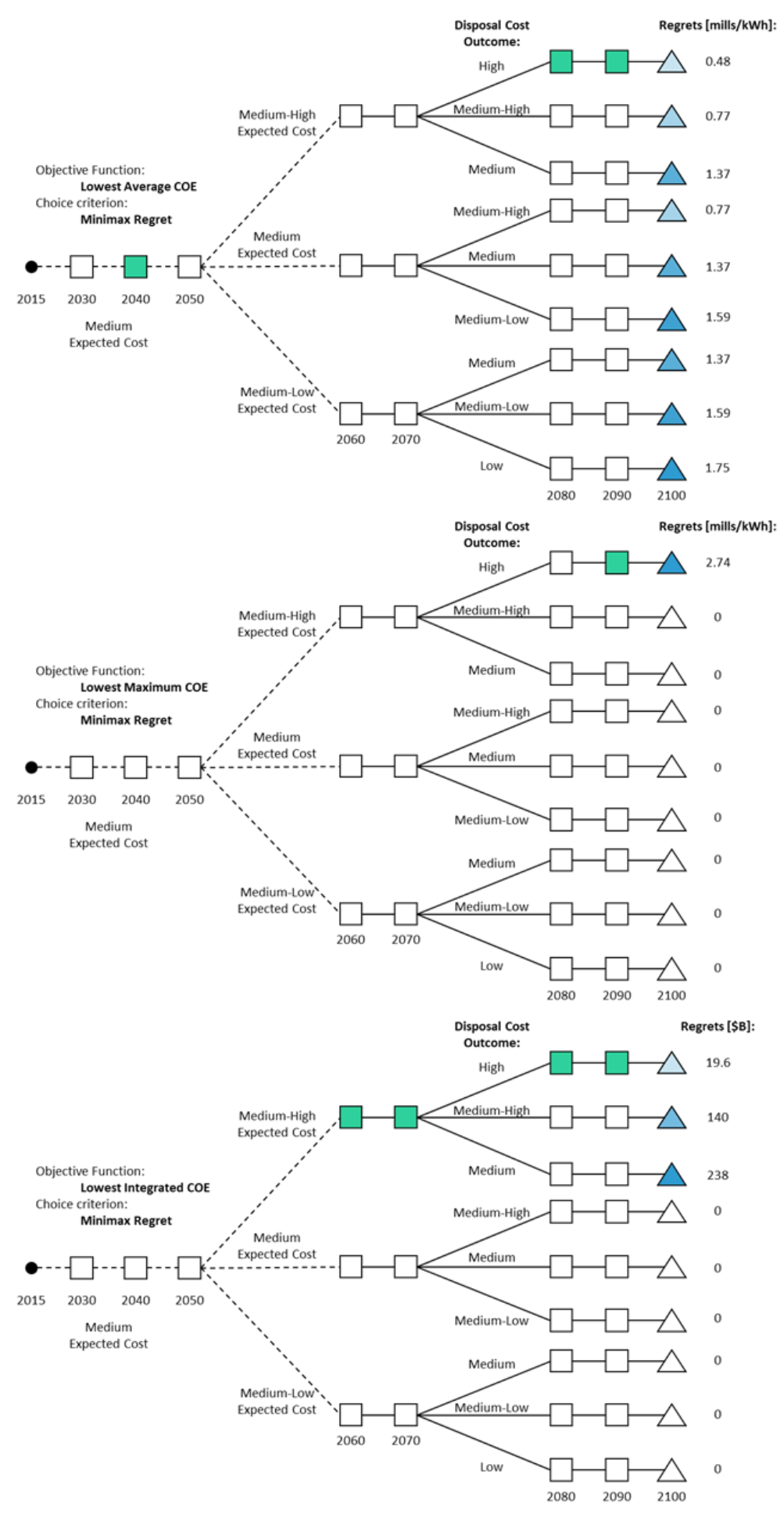

Fig. 4.5a. Reference scenario optimized transition for minimax regret choice criterion. Green squares indicate construction of a reprocessing unit; white squares indicate foregone build opportunity. 


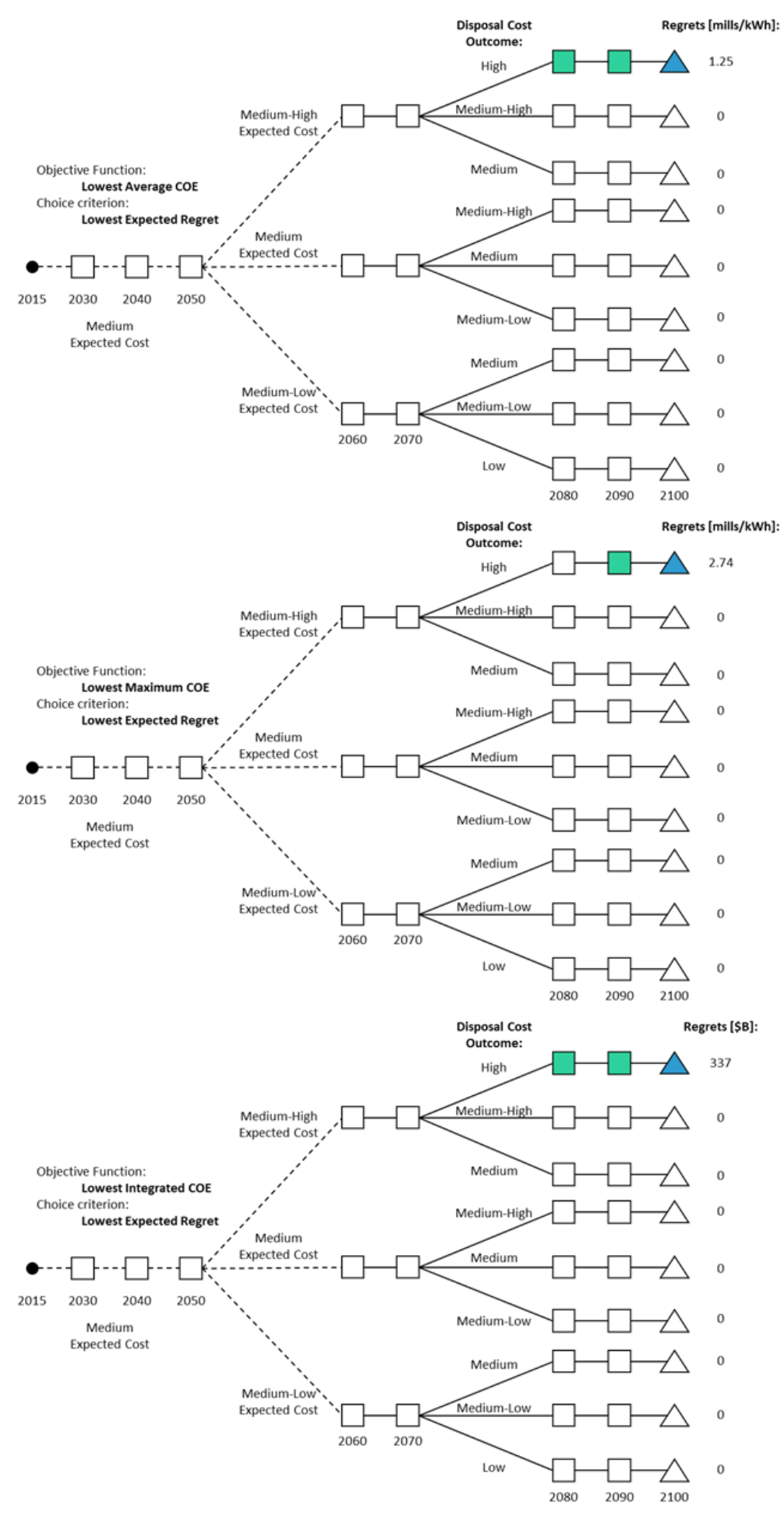

Fig. 4.5b. Reference scenario optimized transition for lowest expected regret choice criterion. Green squares indicate construction of a reprocessing unit; white squares indicate foregone build opportunity. 
Only when the lowest average LCOE objective function and minimax choice criterion are used is reprocessing capacity added in stage 2 (2030 to 2050). Likewise, reprocessing capacity is only added in stage 3 (2060 and 2070 build dates) when the lowest integrated LCOE objective function and minimax choice criterion are applied. In both cases, the minimax choice criterion is shown to be more conservative than the expected regret choice criterion by choosing to build more reprocessing units and earlier in the simulation as a hedge against the high disposal cost outcome. However, if the high disposal cost does not transpire, the transition is abandoned. If the expected regret choice criterion is applied in these cases, agents instead choose to wait for more information in 2070, as they are hedging against the expected outcome, as opposed to the worst-case scenario.

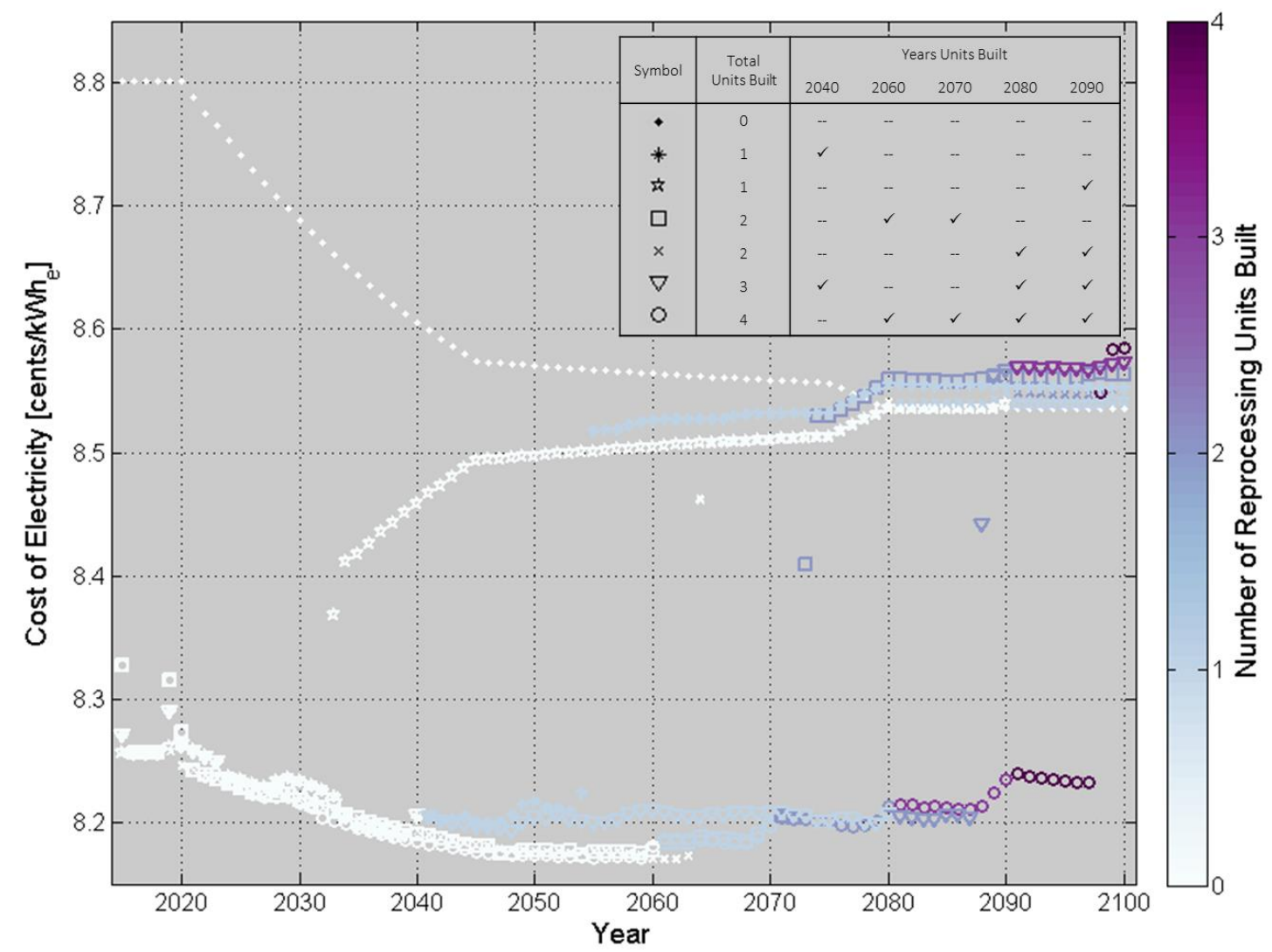

Fig. 4.6. Annual LCOE for optimal hedging strategies and high disposal cost end-state. 
The annual LCOE and FR generating capacity for each of the strategies shown in Fig. 4.5 are given in Fig. 4.6 and Fig. 4.7, respectively. Again, the strategies selected using the lowest average and integrated LCOE objective functions have a lower LCOE through a majority of the decision-making period as compared to the strategy selected using the lowest maximum LCOE objective function. In all cases, the strategies selected have insufficient reprocessing capacity to reprocess all UF generated in this period. However, the strategy selected using the lowest maximum LCOE objective function allows only a small number of FRs (Fig. 4.7) to be built, keeping the LCOE lower when the changeover to direct disposal occurs. This is reflected in Fig. 4.1c where strategies that build less reprocessing capacity and later in the simulation benefit in terms of this objective function.

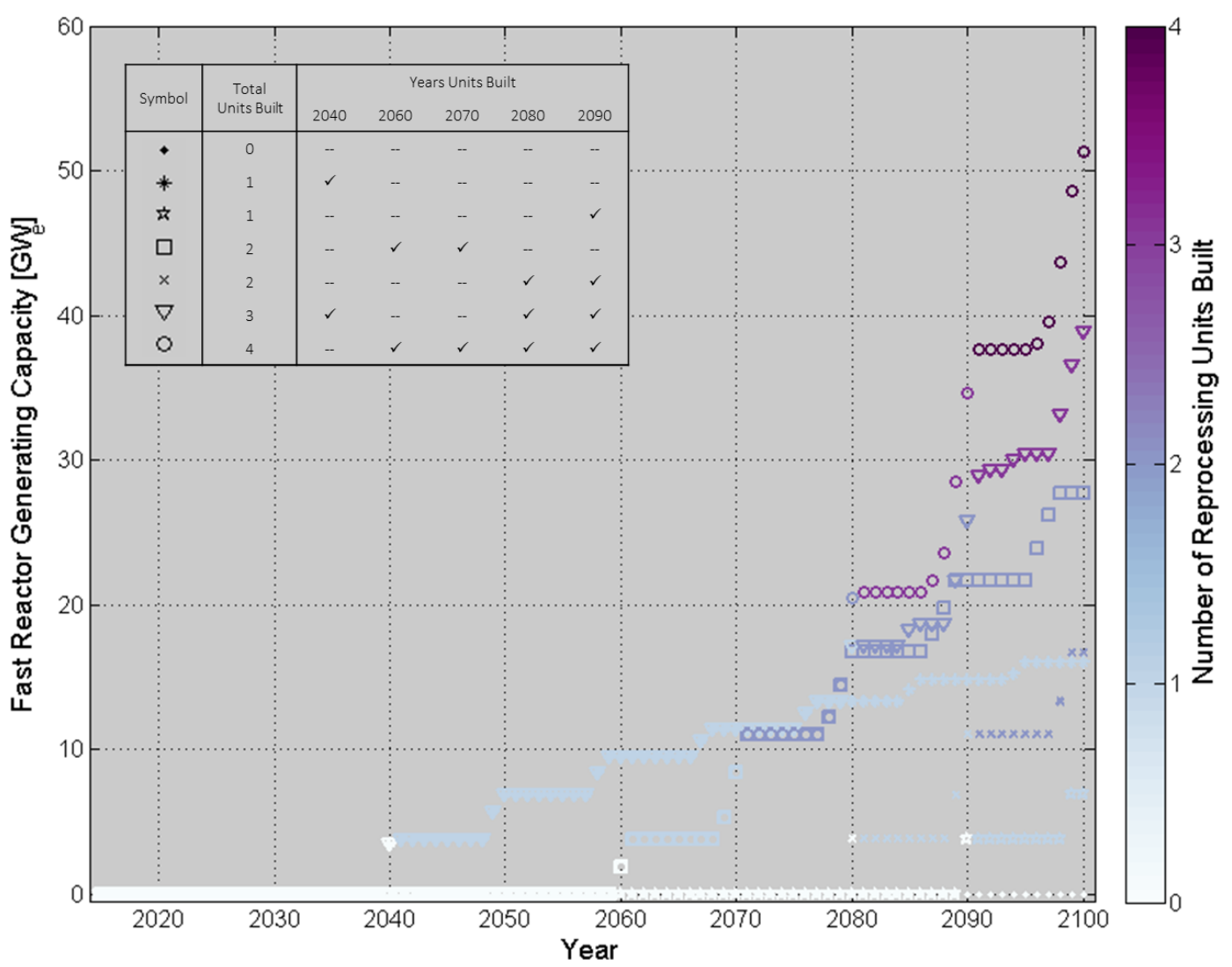

Fig. 4.7. Annual FR generating capacity $\left(\mathrm{GW}_{\mathrm{e}}\right)$ for optimal transition strategies. 


\subsection{Sensitivity Study: RATIO BeTWEen HLW AND SNF Disposal CostS}

A significant factor affecting the benefit of transitioning toward continuous recycle in FRs is the ratio between the HLW and SNF disposal costs. HLW contains FPs and trace amounts of TRU which is dependent on the efficiency of the recycle process. At equilibrium, recycle in FRs may achieve a factor of 10 decrease in the volume of HLW (NEA, 2006) and factor of 50 decrease in the long-term decay heat production (Dixon et al., 2008), however this may be entirely offset if the cost of disposing HLW on a per-kg IHM basis is much higher than the cost of disposing SNF. Engineering estimates of the cost of HLW and SNF disposal from (Shropshire et al., 2009) are given in Table 4.3, with their calculated ratios. Lower HLW disposal costs may be achieved through higher FP loading of the HLW waste form or a higher heat loading of the geologic repository. For this sensitivity analysis, a ratio of 3 for the HLW to SNF disposal costs is used for illustration purposes. The resulting HLW and SNF disposal costs for the five end-states are given in Table 4.4; all other factors are kept constant from the reference scenario, discussed in Section 4.1.

Table 4.3. SNF and HLW disposal cost estimates from (Shropshire et al., 2009)

\begin{tabular}{|c|c|c|c|}
\hline \multicolumn{4}{|c|}{ Disposal Costs } \\
\hline & $\begin{array}{c}\text { SNF } \\
(\$ / \mathrm{kg} \mathrm{IHM})\end{array}$ & $\begin{array}{c}\text { HLW } \\
(\$ / \mathrm{kg} \text { IHM in HLW) }\end{array}$ & $\begin{array}{c}\text { Ratio } \\
\text { (HLW:SNF) }\end{array}$ \\
\hline Low & 400 & 1,625 & 4.06 \\
\hline Nominal & 650 & 6,500 & 10.0 \\
\hline High & 1000 & 8,125 & 8.13 \\
\hline
\end{tabular}

Fig. 4.8 gives the annual LCOE for each reprocessing capacity expansion profile and the medium-low and high SNF and HLW disposal cost outcomes; the results from these two end-states (Fig. 4.1a and c) from the reference scenario are also given for comparison purposes. The overall distribution and trends of the LCOE data do not differ dramatically from those seen in the reference scenario, however, the cost of the 
reprocessing-based fuel cycles is lowered due to cheaper HLW disposal. This is most readily seen when contrasting Fig. $4.8 \mathrm{~b}$ and d. Here, the cost of the reprocessing-based fuel cycle is approximately 5 percent lower than in the reference case scenario, and when transition to the direct disposal fuel cycle occurs, costs are not elevated as much due to the existing FR infrastructure.

Table 4.4. SNF and HLW Disposal Costs used in Section 4.2 sensitivity analysis

\begin{tabular}{r|cc} 
& \multicolumn{2}{c}{ Disposal Costs } \\
End-states & $\begin{array}{c}\text { SNF } \\
(\$ / \mathrm{kg} \mathrm{IHM})\end{array}$ & $\begin{array}{c}\text { HLW } \\
(\$ / \mathrm{kg} \text { IHM in HLW })\end{array}$ \\
\hline Low & 400 & 1,200 \\
Medium-Low & 650 & 1,950 \\
Medium & 1,000 & 3,000 \\
Medium-High & 1,950 & 5,850 \\
High & 6,500 & 19,500
\end{tabular}

Despite a lower HLW to SNF disposal cost ratio than in the reference scenario, the perfect information strategies for 3 out of the 5 end-states (low, medium-low, and medium) is again to directly dispose LWR fuel and forego building any reprocessing facilities. However, for 2 out of the 5 end-states (the medium-high and high end-states), different perfect information strategies arise, see Fig. 4.9. Fig. 4.10 gives the annual LCOE for the strategies shown in Fig. 4.9. For the medium-high HLW and SNF disposal cost outcome and the lowest average and maximum LCOE objective functions, the perfect information strategy calls for the construction of $21,000 \mathrm{tIHM} / \mathrm{yr}$ reprocessing units. However, the lowest integrated LCOE objective function still suggests no transition be made, due to a marginal increase in the integrated LCOE when even 2 units are built late in the simulation. For the high HLW and SNF disposal cost outcome, the perfect information strategies seek to build 4 reprocessing units. Again, with the lowest maximum LCOE objective function, 
for the majority of the simulation a larger annual LCOE is realized, which is reflected in the higher average and integrated LCOE.
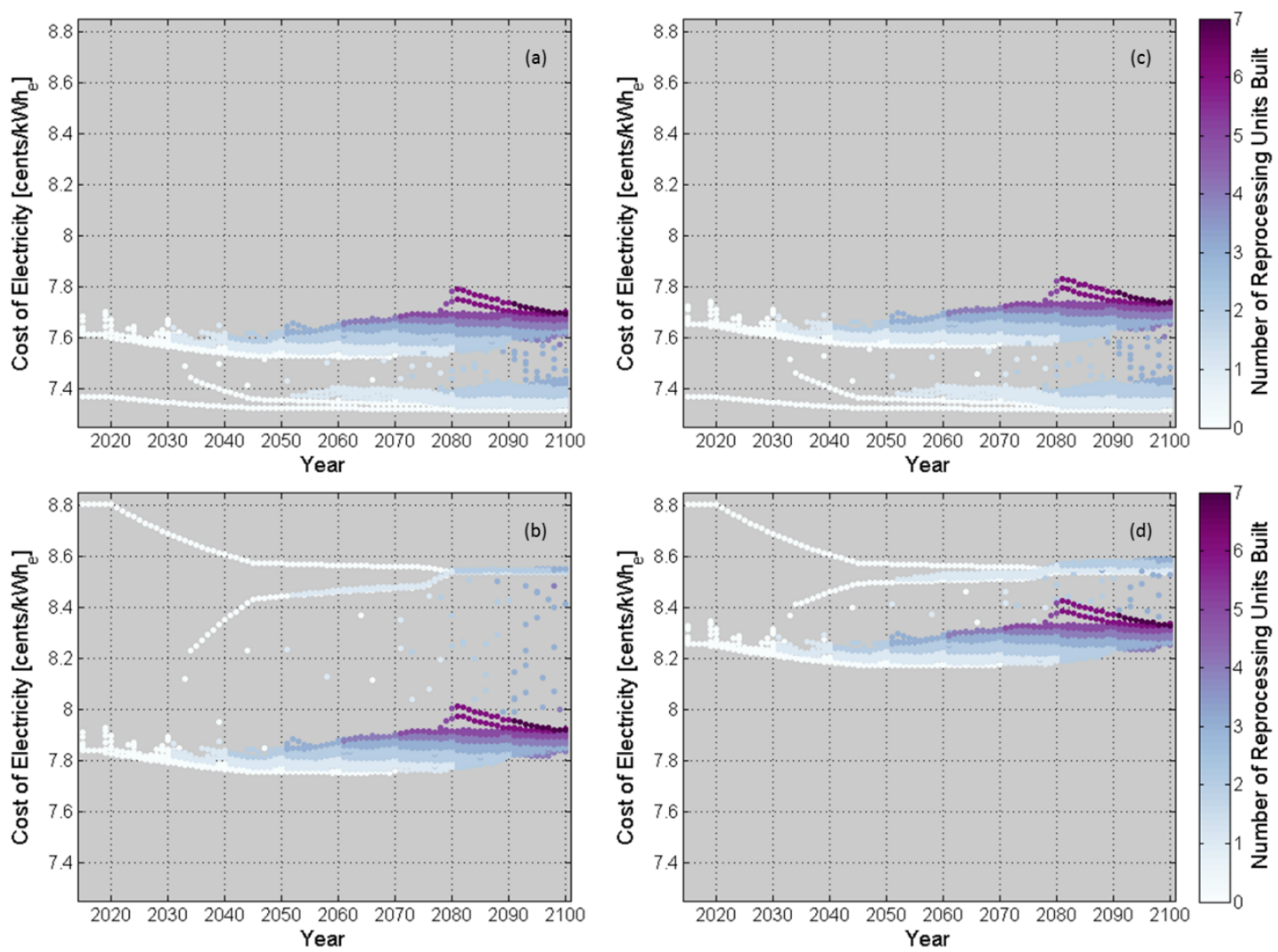

Fig. 4.8. Annual LCOE for all reprocessing capacity expansion profiles and (a) mediumlow and (b) high end-states. Reference scenario (c) medium-low and (d) high end-states shown for comparison.

The optimal hedging strategies for each objective function and choice criterion pair are shown in Fig. 4.11, along with the regrets accrued by the hedging strategies selected and the end-states realized. The strategies identified using the minimax regret choice criterion call for the construction of a single (lowest average and integrated LCOE objective functions) or two (lowest maximum LCOE objective function) before the revelation of addition cost information in 2050. However, if the information in 2050 suggests a medium-low or medium expected disposal cost, the transition to the 
reprocessing-based fuel cycle is abandoned until the 2070 resolution date. Then, if the medium-high disposal cost outcome is realized, the transition may be continued (if the lowest average or integrated LCOE objective function is applied).

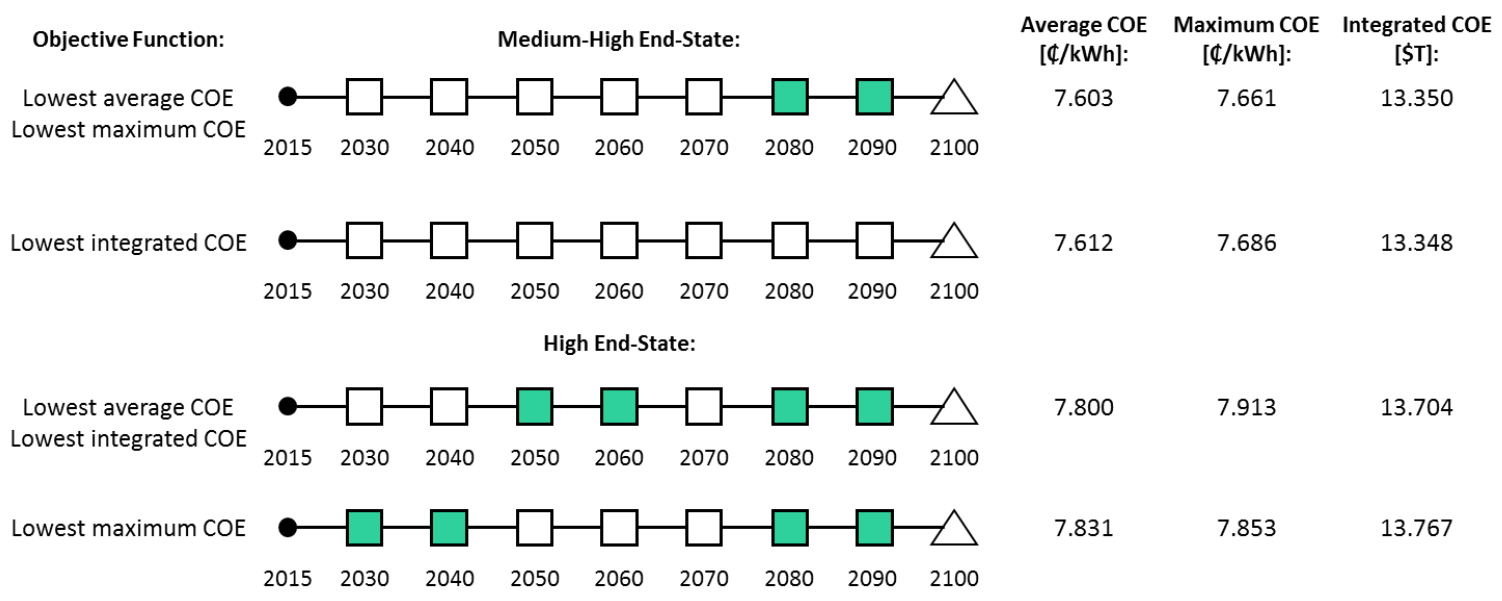

Fig. 4.9. Perfect information strategies for medium-high and high disposal cost outcomes. Green squares indicate construction of a reprocessing unit; white squares indicate foregone build opportunity.

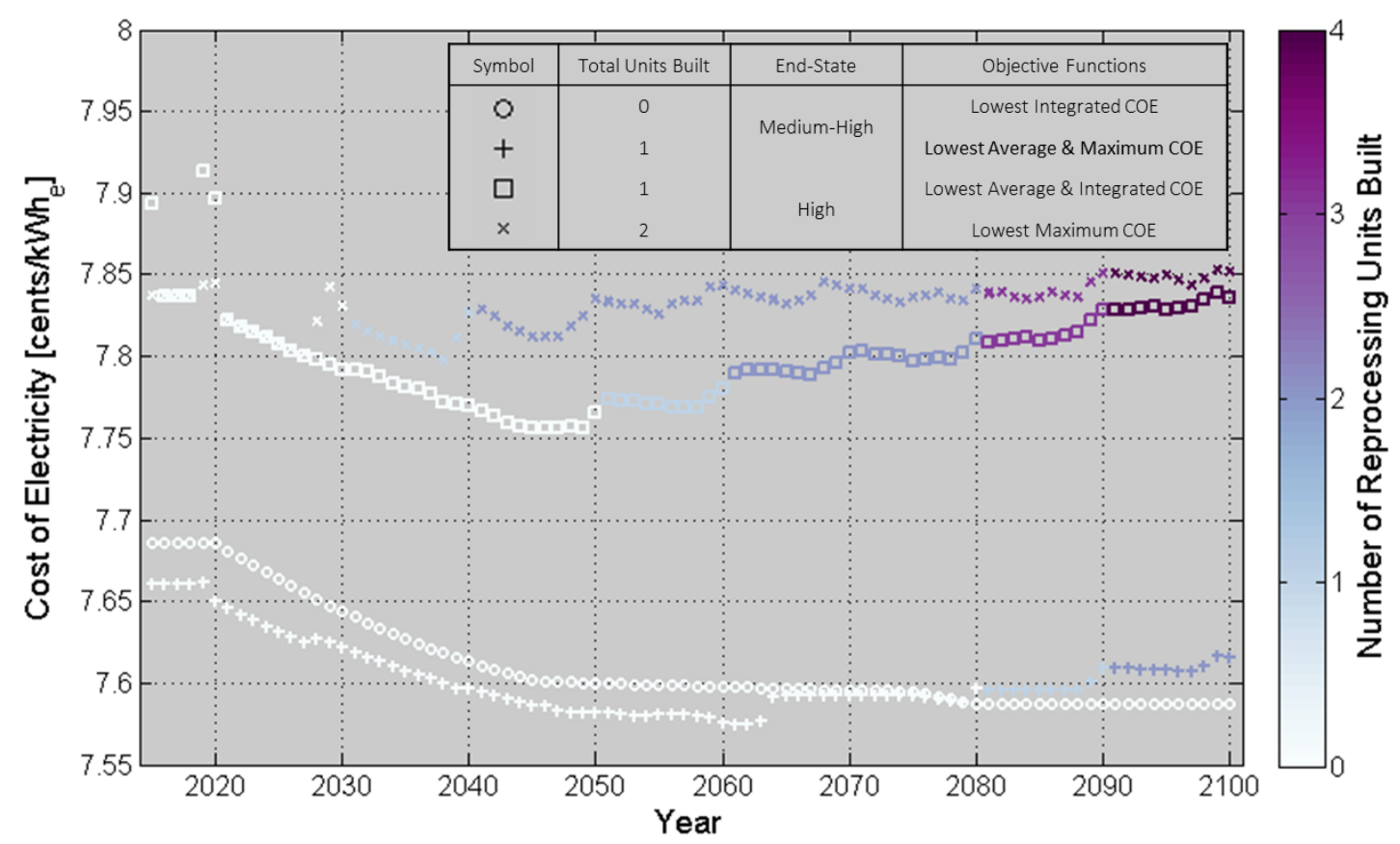

Fig. 4.10. LCOE for perfect information strategies corresponding to medium-high and high end-states. 
Strategies identified using the expected regret choice criterion delay building any reprocessing units until more information is available in 2050 . Here the collective $2 / 3$ probability of the low, medium-low and medium end-states occurring influences the decision to delay building any reprocessing facilities before more information is available. Generally, a similar number of total units built is the same between the two choice criteria. However, there are two major exceptions. First, for the low, medium-low and medium endstates, no units are built when the expected regret choice criterion is applied, whereas for the minimax regret choice criterion 1-2 units are built early in the simulation as a hedge against the medium-high and high end-states. Second, when the expected regret choice criterion is applied with the lowest maximum choice criterion, the transition to the reprocessing-based fuel cycle is delayed until 2080 when two units are built (medium-high end-state) or until 2090 when a single unit is built (high end-state), whereas when the minimax regret choice criterion is applied, the transition begins in 2050 . Notice that post2070, the strategies presented are no longer hedging strategies, but simply perfect information strategies since the SNF and HLW disposal costs are made known in 2070. Choosing to delay the transition until 2090, as opposed to building 2 reprocessing units in 2080 and 2090, increases the regrets incurred by approximately 30 percent. However, due to the chosen objective function, agents choose to delay building until 2090. In this situation, the lowest maximum LCOE objective function may be overly sensitive to aberrations in the annual LCOE.

This sensitivity study indicates that a lower HLW to SNF disposal cost ratio (relative to the reference scenario) is required to begin a transition towards continuous recycle in FRs in stage 2 (between 2030 and 2050). Following 2070, the transition may be abandoned if the low, medium-low, or medium end-state is realized, or accelerated if the medium-high or high end-state is realized. 


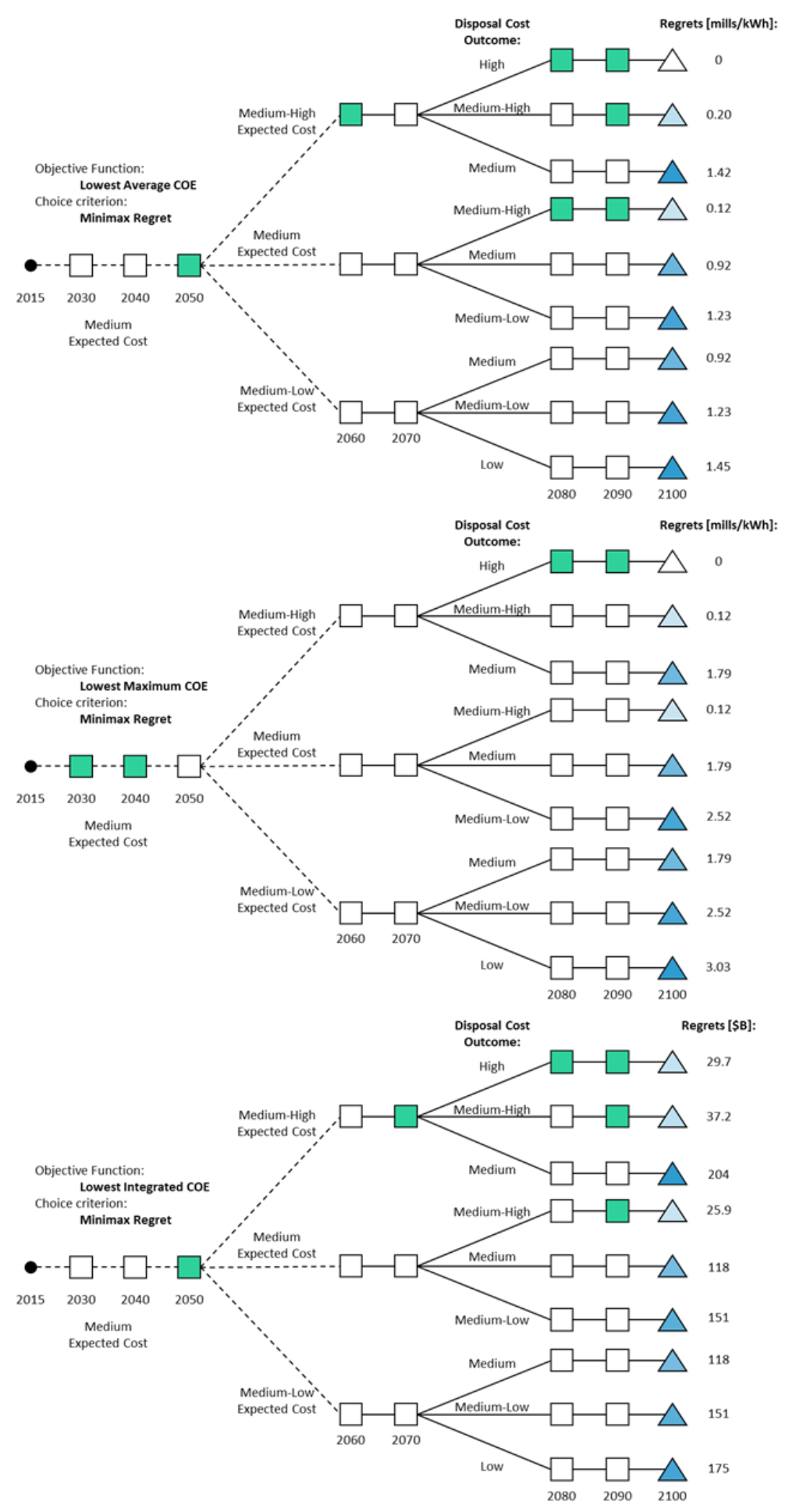

Fig. 4.11a. Section 4.2 scenario optimized transition for minimax regret choice criterion. Green squares indicate construction of a reprocessing unit; white squares indicate foregone build opportunity. 


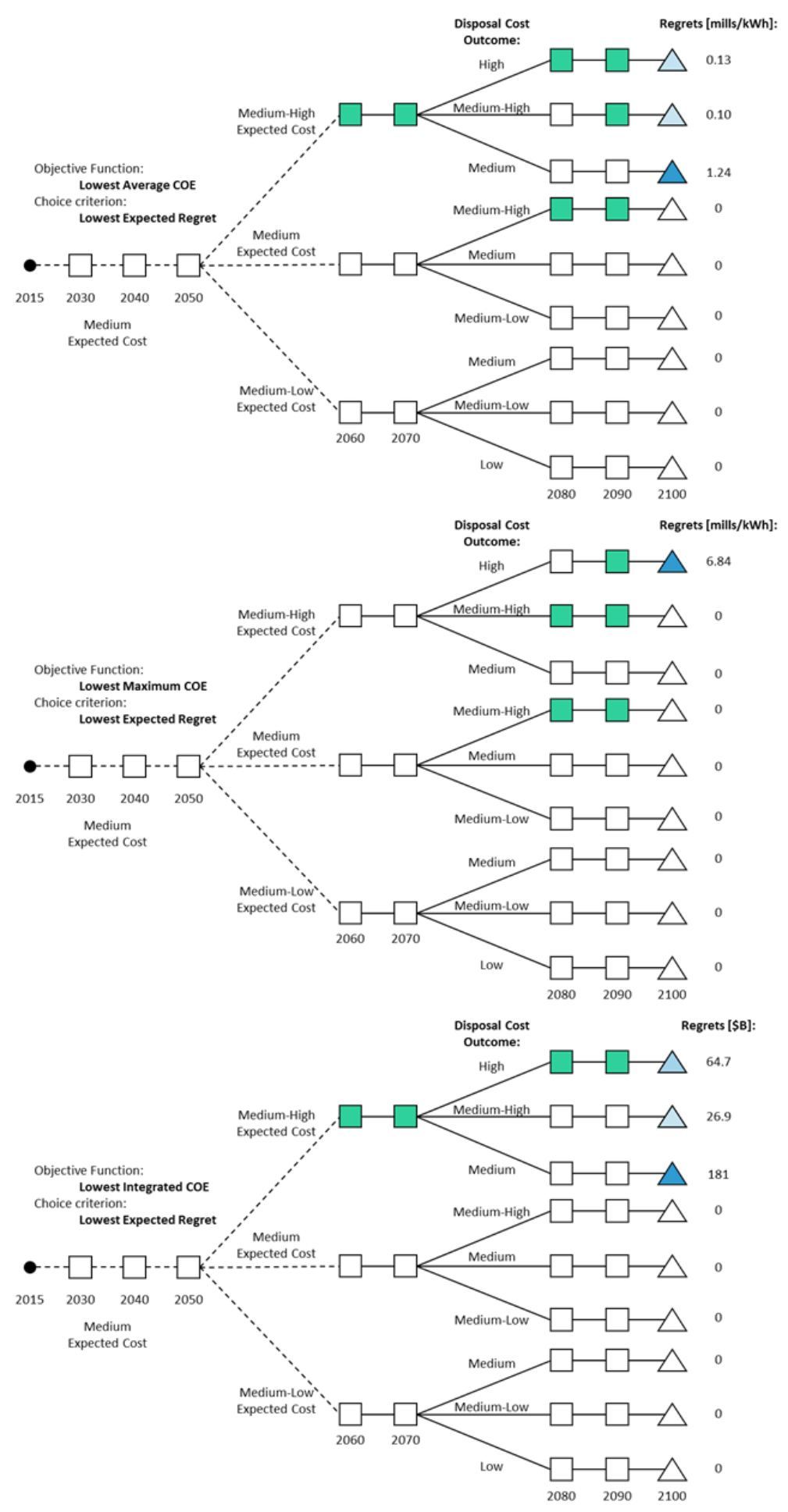

Fig. 4.11b. Reference scenario optimized transition for lowest expected regret choice criterion. Green squares indicate construction of a reprocessing unit; white squares indicate foregone build opportunity. 


\subsection{Sensitivity Study: FASt Reactor Capital CoST}

Reactor capital costs account for a significant portion of the LCOE in the nuclear fuel cycle. In the reference scenario, when the direct disposal strategy is employed with a medium-low SNF disposal cost, reactor capital costs account for nearly 80 percent of the LCOE. As such, the relative difference in reactor capital costs between competing reactor types heavily influence which technology is selected. Engineering estimates of the overnight capital cost of LWRs and FRs from (Shropshire et al., 2009) are given in Table 4.5. This sensitivity analysis employs the low FR capital cost of $3,000 \$ / \mathrm{kW}$; all other factors are kept constant from the reference scenario, discussed in Section 4.1. Nominal values for both LWRs and FRs were used in the reference scenario.

Table 4.5. LWR and FR overnight capital cost estimates from (Shropshire et al., 2009)

\begin{tabular}{|c|c|c|}
\hline & $\begin{array}{l}\text { LWR Overnight Capital Cost } \\
\qquad\left(\$ / k W_{\mathrm{e}}\right)\end{array}$ & $\begin{array}{l}\text { FR Overnight Capital Cost } \\
\left(\$\left(\$ / k W_{e}\right)\right.\end{array}$ \\
\hline Low & 2,300 & 3,000 \\
\hline Nominal & 3,500 & 4,200 \\
\hline High & 5,000 & 7,000 \\
\hline
\end{tabular}

Fig. 4.12a-c gives the annual LCOE for each reprocessing capacity expansion profile and the medium-low, medium-high and high SNF and HLW disposal cost outcomes; Fig. 4.12d-f repeat the results from these three end-states (Fig. 4.1a-c) from the reference scenario for comparison purposes. A clear difference is seen in the trends of Fig. 4.12a-c and Fig. 4.12d-f. Here, the baseline costs for both the reprocessing-based and direct disposal fuel cycles are the same across any given end-state. However, in Fig. 4.11a-c the LCOE decreases later in the simulation once FRs are built, since FR capital costs are lower than LWR capital costs. Again, the construction of 6 or 7 reprocessing units may lead to elevated costs due to underutilized capacity, but to a lesser degree than in the reference scenario. 

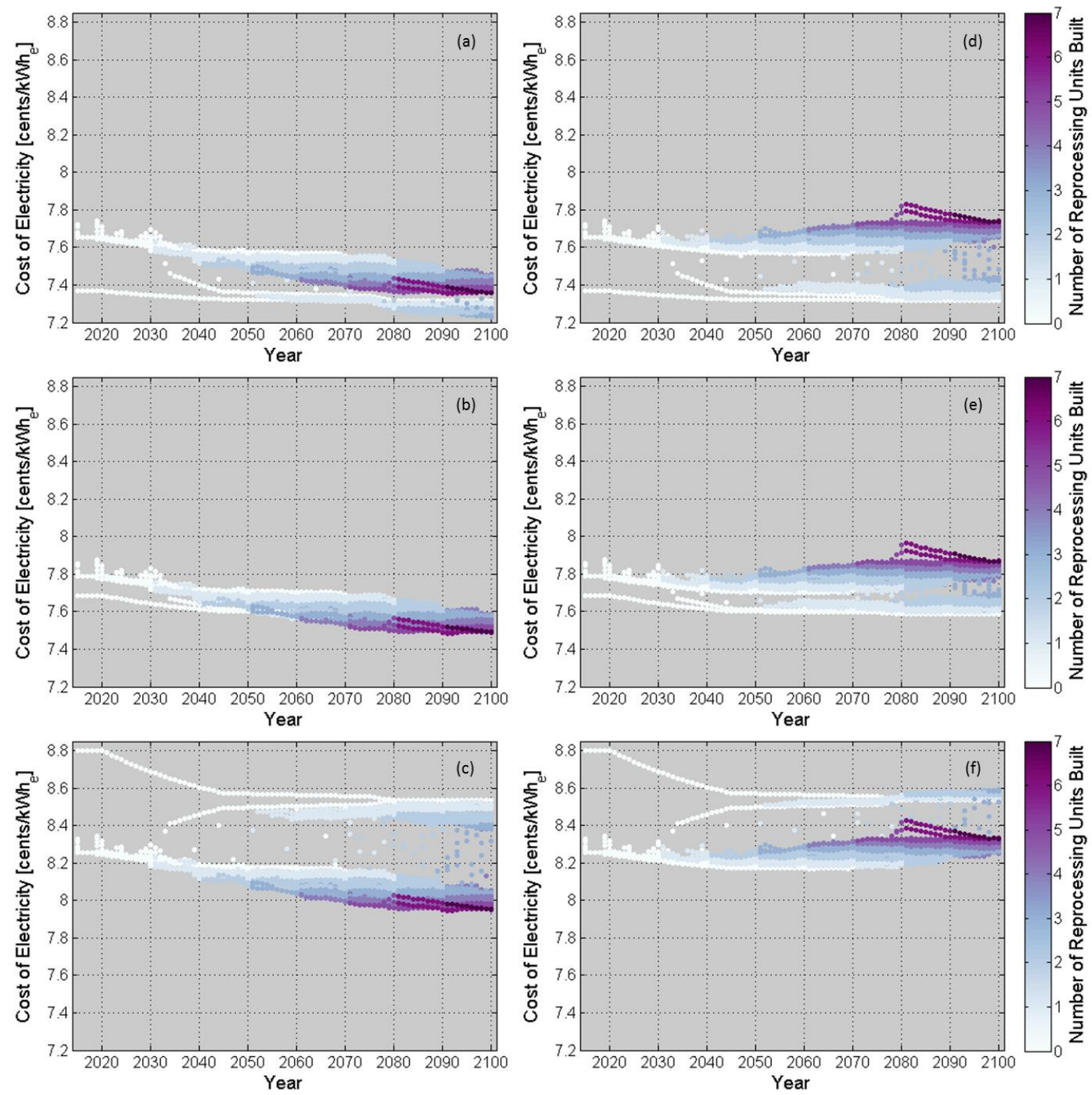

Fig. 4.11. Annual LCOE for all reprocessing capacity expansion profiles and (a) mediumlow, (b) medium-high and (c) high end-states. Reference scenario (d) medium-low, (e) medium-high and (f) high end-states shown for comparison

Fig. 4.13 gives the perfect information strategies for the medium-high and high end-states; the perfect information strategies for the low, medium-low and medium end-states are again the direct disposal strategies. Fig. 4.14 gives the annual LCOE for the strategies shown in Fig. 4.13. For the medium-high end-state, the perfect information strategy either calls for an aggressive transition (lowest average and integrated LCOE 
objective functions, with 6 1,000 tIHM/yr LWR UF reprocessing facilities built) or no transition at all (lowest maximum LCOE objective function). In the case when the lowest maximum LCOE objective function is applied, the added cost of reprocessing early in the simulation ensures that the transition never be selected (see Fig. 4.14). This results from assessing costs at the year fuel is in reactors producing electricity; reprocessing costs are ascribed to UF generated from earlier operating LWRs, before any benefit from transitioning to cheaper FRs may be seen. For the high end-state and the lowest average and integrated LCOE objective functions, the same transition strategy is recommended as in the case of the medium-high end-state. While the lowest maximum LCOE objective function suggests 4 reprocessing units be built, this strategy actually has the same maximum LCOE as the strategy identified by the lowest average and integrated LCOE objective functions. The algorithm used to find the maximum LCOE then finds the next maximum (see Fig. 4.14), resulting in the strategy shown in Fig. 4.13.

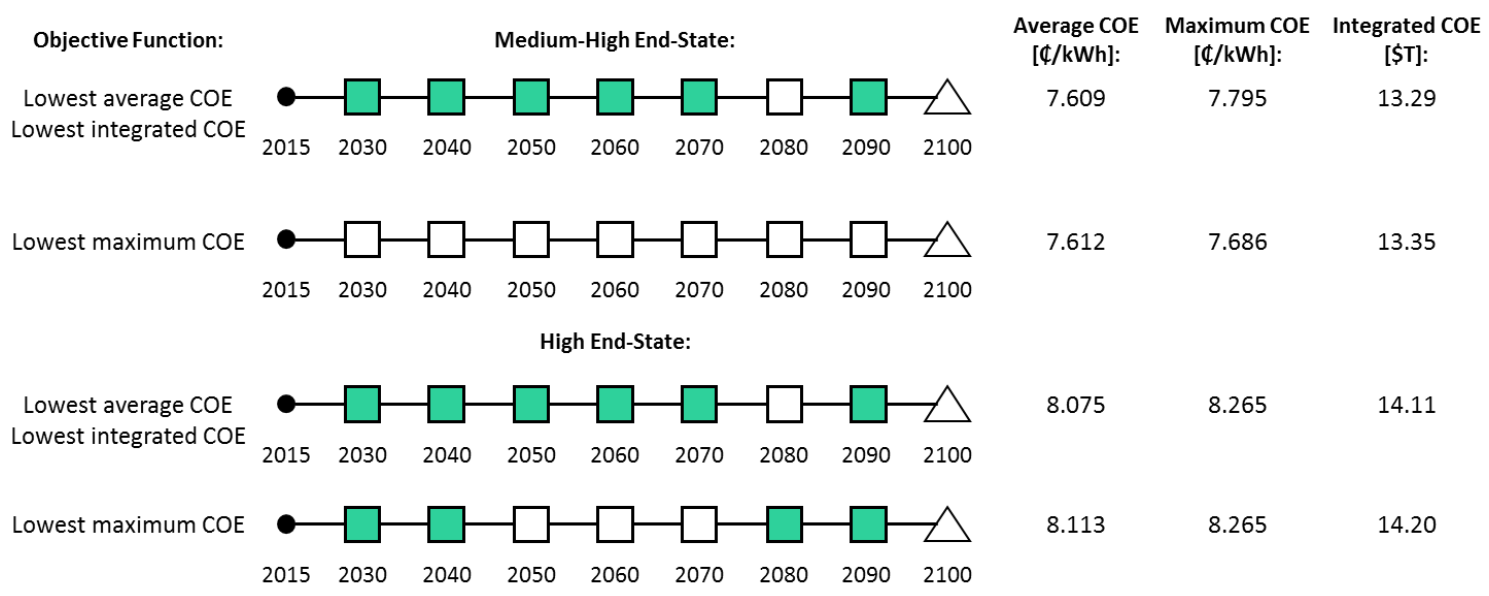

Fig. 4.13. Perfect information strategies for medium-high and high disposal cost outcomes. Green squares indicate construction of a reprocessing unit; white squares indicate foregone build opportunity. 


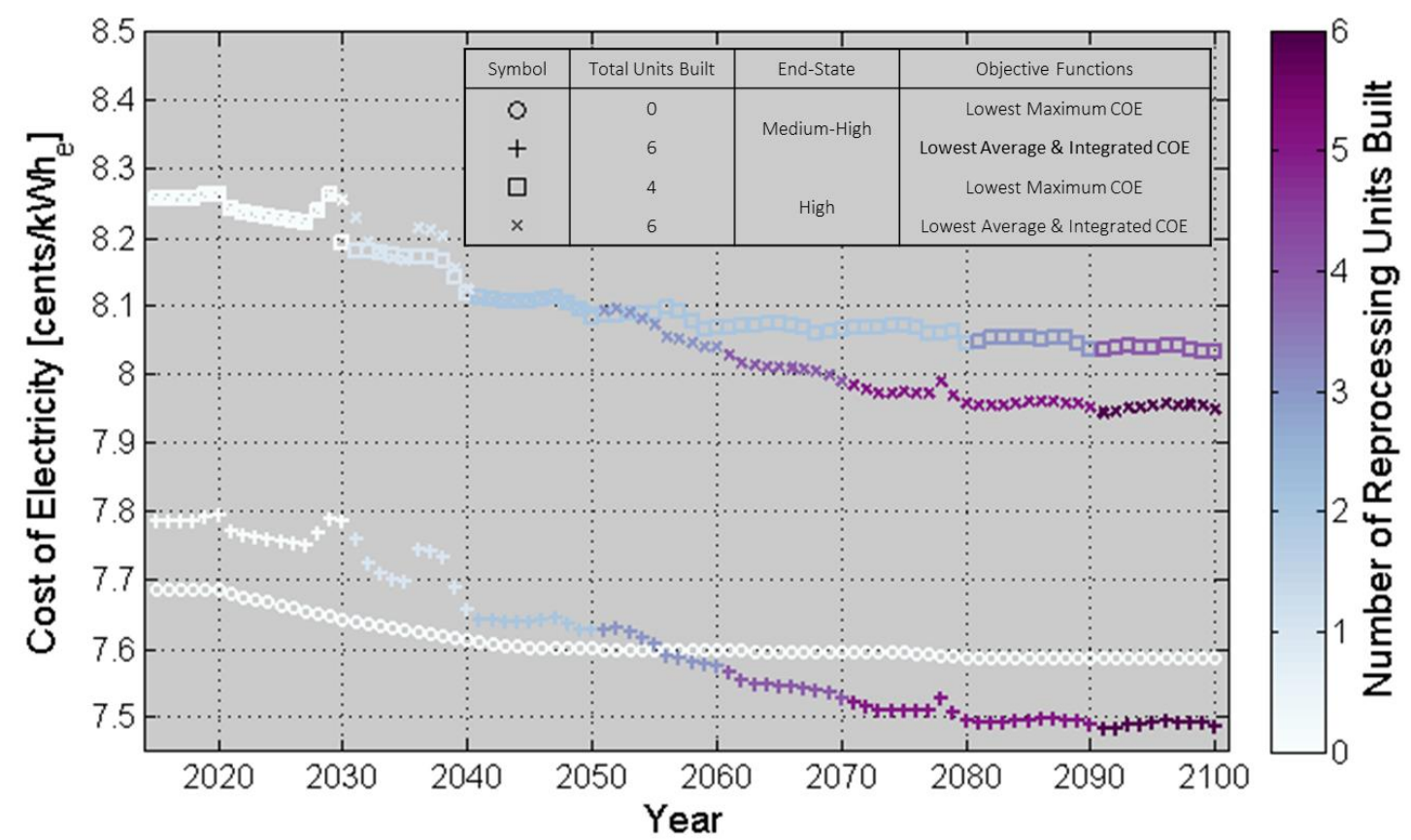

Fig. 4.14. LCOE for perfect information strategies corresponding to medium-high and high end-states.

The optimal hedging strategies for each objective function and choice criterion pair are shown in Fig. 4.15, along with the regrets accumulated by the hedging strategies selected and the end-states realized. Regardless of which choice criterion is applied, when the lowest integrated LCOE objective function is applied, 6 reprocessing units are built. Here, the regrets seen when no transition is started early in the simulation and either the medium-high or high end-state is realized dominate. Then, since FRs are sufficiently less expensive compared to LWRs, more reprocessing units are built later in the simulation to support more FR builds. The same situation happens when the lowest average LCOE objective function is applied, but only with the minimax regret choice criterion. Significantly fewer reprocessing units are built when the expected regret choice criterion is used with this objective function, since the $2 / 3$ probability of the low, medium-low and medium end-states dominates. 
For cases where the lowest maximum LCOE objective function is applied, regardless of the choice criterion selected, the transition is delayed until after the 2070 resolution date, and only began if the high end-state is realized. In terms of the lowest maximum LCOE objective function, agents benefit greatly by employing a wait-and-see strategy. Generally, the added costs of reprocessing are sufficient to ensure that strategies where the transition is started early are not selected (see Fig. 4.11a-b). However, if the high end-state is realized in 2070, agents may build reprocessing units in 2080 and 2090 and still benefit from moving towards the reprocessing-based fuel cycle. This results since costs are assessed the year that fuel is discharged. Fuel discharged in 2015 may be reprocessed later in 2080 when a reprocessing unit is built, and benefit from the cheaper disposal cost if the high end-state is realized (Fig. 4.11c).

This sensitivity study indicates that if FR capital costs are sufficiently low, it is advantageous to begin an aggressive transition to the reprocessing-based fuel cycle as soon as possible regardless of the cost of disposal. Aside from results seen when the lowest maximum LCOE objective function is applied, agents do not truly hedge unless the lowest average LCOE objective function and lowest expected regret choice criterion are applied. For more expensive FR capital costs than those assumed in this sensitivity study, the strategy is consistently to wait as long as possible before starting to build reprocessing capacity. 


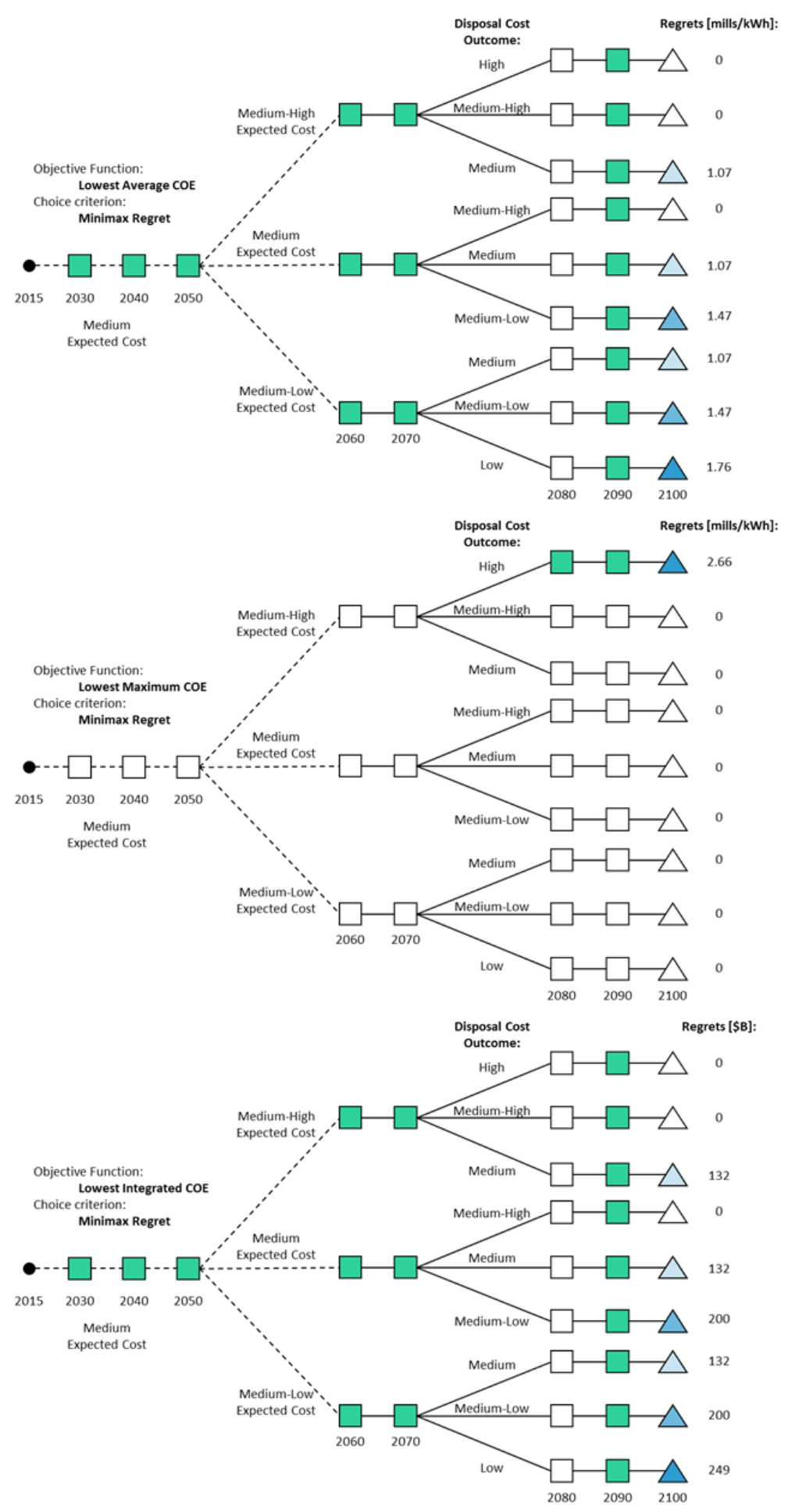

Fig. 4.13a. Section 4.2 scenario optimized transition for minimax regret choice criterion. Green squares indicate construction of a reprocessing unit; white squares indicate foregone build opportunity. 


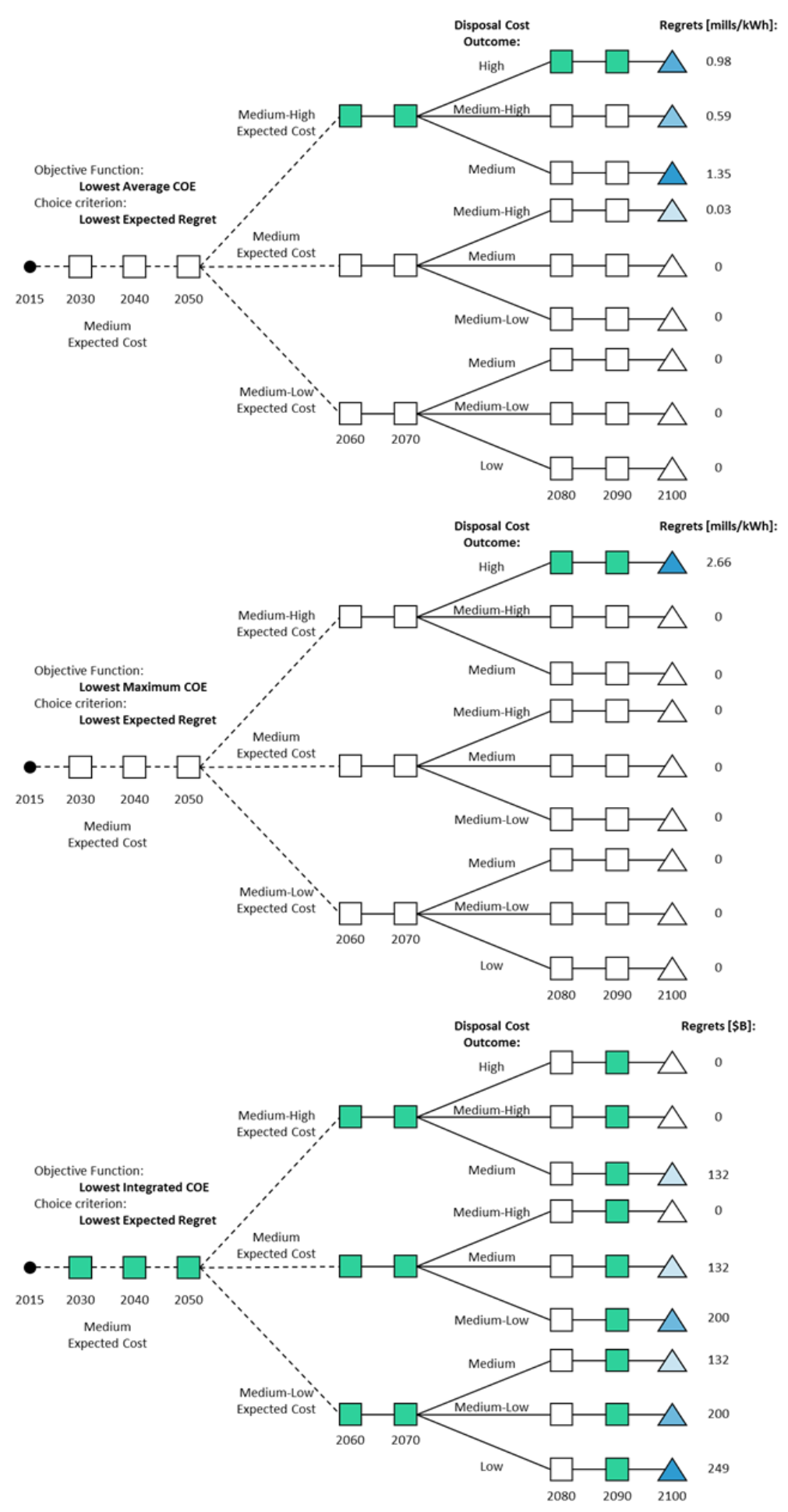

Fig. 4.13b. Reference scenario optimized transition for lowest expected regret choice criterion. Green squares indicate construction of a reprocessing unit; white squares indicate foregone build opportunity. 


\section{Chapter 5: Conclusion}

The work presented in this thesis has demonstrated a new methodology for handling uncertainty in fuel cycle transition analyses. A case study involving transition from the current once-through LWR fuel cycle to one relying on continuous recycle in FRs was presented. The transition is subject to uncertainty in the cost of SNF and HLW disposal in a geologic repository, set to open some years into the future. This transition scenario was cast as a no-data decision problem, where it is impossible to gain information about the state of the uncertain parameters that will ultimately prevail. In general, for this scenario and two sensitivity analyses involving varying the ratio between HLW and SNF disposal costs and FR capital costs, the transition suggested using the decision analytic framework is only a partial closing of the fuel cycle prior to the repository open date, when uncertainties are resolved. After information is made available, agents may choose to alter their strategy and continue or abandon the transition.

The effect of objective functions (for selection of perfect information strategies) and choice criterion (for selection of hedging strategies) on the optimal strategies chosen was also examined. Generally, the lowest average and integrated LCOE objective functions perform similarly. However, the lowest maximum LCOE objective function appears overly sensitive to aberrations in the annual LCOE that arise due to idle capacity. This sensitivity leads to selection of strategies that perform poorly over a majority of the simulation as compared with the lowest average or integrated LCOE, except for a single year (or two) in the simulation. Because of this sensitivity, use of the lowest maximum LCOE objective function is not recommended for this application, but may sometimes be desirable when a risk-averse agent seeks to avoid a worst-case outcome despite its likelihood. The minimax regret choice criterion is shown to be the more conservative choice criterion over the lowest 
expected regret choice criterion, as it is independent of the probability distribution on the outcomes. The minimax regret choice criterion instead acts to hedge against the worst-case outcome, instead of the expected outcome.

Some limitations of the work presented here are discussed below:

- The work presented in this thesis assumes perfect predictions (North, 1968). In essence, the results found using the VEGAS fuel cycle simulator are only as good as the simulator itself. However, no fuel cycle simulator or energy-economic model is fully capable of encompassing all factors involved in technological change, especially the effects of policy and technological spill-overs (Weyant and Olavson, 1999).

- While the methodology presented allows for explicit handling of imperfect information, it still places burden on the modeler to predict which states of nature may prevail. The results are then heavily reliant on realistic depictions of the future. Conclusions drawn from this analysis only include hedging against the outcomes which the modelers see as reasonably plausible. However, use of the minimax regret choice criterion may act to allay this burden as it only requires identification of a worst-case outcome.

- The method for applying the decision-analysis in this thesis may prove prohibitively expensive if the number of options considered is increased significantly. Given the "brute force" approach taken in this thesis, where each option is directly enumerated using a systems model of the nuclear fuel cycle, the number of computations required scales by $n^{m}$, where $n$ is the number of end-states for an uncertain parameter and $m$ is the number of parameters. Alternatively, stochastic optimization may introduce avenues for decreasing the number of computations required. Specifically, randomized search methods may be used to 
quickly search the parameter space. The stochastic optimization methodology may be implemented in future work as a look-ahead tool with a light-weight model of the nuclear fuel cycle (similar to the VEGAS tool) for further analysis by a more sophisticated fuel cycle simulator. Regardless, the number of metrics considered (different objective functions and choice criteria) have no bearing on the number of computations required since these are determined following completion of said computations.

- The results presented represent decisions made if a single agent were acting with a long-term planning horizon, similar to a government entity. However, many agents act with a more short-sighted planning horizon. The issue of limited foresight may be the subject of future work. Here, simulations are optimized within the visible planning horizon of say 10 years from the beginning of the simulation. Then, the state of the system at the end of the planning horizon is saved, and used to initialize the optimization problem for the next 10 years. This process is repeated until the end of the simulation. 


\section{APPENDIX A: VEGAS SIMULATION INPUTS}

The primary simulation inputs for the VEGAS simulations described in Section 3 are documented here.

Table A.1 gives initial reactor fleet data and the electricity demand profile for the simulation time period. Table A.2 lists reactor power parameters for each reactor appearing in the reference VEGAS simulation, as well as a reactor fuel recipe. Reference reactor overnight capital costs are given in Table A.3 with their corresponding table look-ups in (Shropshire et al., 2009).

Table A.1. Electricity generation profile.

\begin{tabular}{|c|c|c|c|c|}
\hline Year & Item & Value & Unit & Reactor Type \\
\hline \multicolumn{5}{|c|}{ A. Initial reactor fleet data } \\
\hline 2015 & Initial Generation Capacity & 100 & $\mathrm{GW}_{\mathrm{e}}$ & - \\
\hline 2020 & Year Initial Fleet Begins Retiring & 2015 & yr & - \\
\hline 2050 & Year Initial Fleet Finishes Retiring & 2045 & yr & - \\
\hline \multicolumn{5}{|c|}{ B. Initial generating capacity } \\
\hline 2015 & Capacity Data & 100 & $\%$ & PWR \\
\hline \multicolumn{5}{|c|}{ C. Exogenous demand for nuclear energy } \\
\hline 2015 & Growth Rate & 2.0 & $\% / y r$ & - \\
\hline \multicolumn{5}{|c|}{ D. Reactor "Try To Build" scenario } \\
\hline 2015 & Try To Build & 100 & $\%$ & PWR \\
\hline 2020 & Try To Build & 100 & $\%$ & APWR \\
\hline \multirow{2}{*}{2030} & \multirow{2}{*}{ Try To Build } & 50 & $\%$ & APWR \\
\hline & & 50 & $\%$ & FR \\
\hline
\end{tabular}


Table A.2. Reactor input parameters

A. Reactor power parameters

\begin{tabular}{lcccc}
\hline & \multicolumn{3}{c}{ Value (Reactor Type) } & \\
& $(\mathbf{P W R})^{\mathrm{a}}$ & $\mathbf{( A P W R}^{\mathrm{b}}$ & $(\mathbf{F R})^{\mathrm{a}}$ & \\
\cline { 2 - 4 } Plant Size $(s)$ & 900 & 1450 & 380 & $\mathrm{MW}_{\mathrm{e}}$ \\
Thermal Efficiency $(\eta)$ & 0.34 & 0.34 & 0.38 & $\frac{\mathrm{MW}_{e}}{\mathrm{MW}_{t}}$ \\
Recirculating Power $(r)$ & 0.02 & 0.02 & 0.02 & $\frac{\mathrm{MW}_{\mathrm{e}} \text { recycled to plant systems }}{\mathrm{MW}_{\mathrm{e}} \text { produced }}$ \\
Discharge Burnup $(\beta)$ & 51 & 60 & 134 & $\frac{\mathrm{MWd}_{t}}{\mathrm{~kg}^{\mathrm{IHM}}}$ \\
Availability $(c)$ & 0.90 & 0.90 & 0.82 & Load factor \\
\hline
\end{tabular}

B. Reactor fuel recipes ${ }^{\mathrm{c}}$

\begin{tabular}{cccc} 
& \multicolumn{3}{c}{ Value (Reactor Type) } \\
& $(\mathbf{P W R})$ & $(\mathbf{A P W R})$ & $(\mathbf{F R})$ \\
\cline { 2 - 4 }$x_{U}$ & 1 & 1 & 0.6661 \\
$x_{P u}$ & 0 & 0 & 0.29571 \\
$x_{M A}$ & 0 & 0 & 0.03819 \\
$y_{U}$ & 0.93439 & 0.92446 & 0.58709 \\
$y_{P u}$ & 0.01198 & 0.01242 & 0.23899 \\
$y_{M A}$ & 0.00100 & 0.00129 & 0.03144
\end{tabular}

${ }^{a}$ From Appendix C in (Dixon et al., 2008).

${ }^{\mathrm{b}}$ From (NEA, 2006).

${ }^{c}$ Fuel recipe from VISION input files; private correspondence J. Jacobson, Idaho National Laboratory. FR fuel recipe taken at equilibrium.

Table A.3. Reactor overnight cost data

\begin{tabular}{lccc} 
Reactor & Value & Unit & Source \\
\hline LWR/ALWR & 3500 & $\$ / k W e$ & Table R1-6 \\
FR & 4200 & $\$ / k W e$ & Table R2-3
\end{tabular}


All unit cost data is provided in Table A.4 and is obtained from (Shropshire et al., 2009) unless otherwise noted. Source in Table A.4 indicates the table look-up for (Shropshire et al., 2009).

Table A.4. Unit cost data

\begin{tabular}{|c|c|c|c|}
\hline Technology & Value & Unit & Source \\
\hline Uranium Mining \& Milling & 75 & $\$ / \mathrm{kg} \mathrm{U}$ as $\mathrm{U}_{3} \mathrm{O}_{8}$ & Table A1-22 \\
\hline Conversion to $\mathrm{UF}_{6}$ & 10 & $\$ / \mathrm{kg} \mathrm{U}$ as $\mathrm{U}_{3} \mathrm{O}_{8}$ & Table B-2 \\
\hline Enrichment & 110 & \$/SWU & Table C1-5 \\
\hline Fuel Fabrication & 250 & $\$ / \mathrm{kg}$ IHM & Table D1-1-3 (for PWR) \\
\hline Transportation to Reactor & 34 & $\$ / \mathrm{kg}$ IHM & Table O2-8 \\
\hline SNF Storage & 300 & $\$ / \mathrm{kg}$ IHM & Table E1-4 \\
\hline SNF Transportation & 92 & $\$ / \mathrm{kg}$ IHM & Table O1-7 \\
\hline Reprocessing $^{\mathrm{a}}$ & 1850 & $\$ / \mathrm{kg}$ IHM & Table F1-4 (UREX +1a) \\
\hline SNF Disposal & 650 & $\$ / \mathrm{kg}$ IHM & Table L-7 \\
\hline HLW Disposal & 6500 & \$/kg IHM in HLW & Table L-8 \\
\hline Separated Actinide Storage & 440 & $\$ / \mathrm{kg} / \mathrm{yr}$ & Table E3-7 \\
\hline $\begin{array}{l}\text { Separated Actinide Vitrification } \\
\text { \& Disposal }\end{array}$ & 5000 & $\$ / k g$ FP & Table G-1 \\
\hline
\end{tabular}

Table F2/D2-7 of (Shropshire et al., 2009) gives a reference unit cost of $\$ 6,000 / \mathrm{kg}$ IHM for integral electrochemical processing of FR metal fuel, including FR fuel fabrication. Little cost data is available in which the two processes are separated. Unit costs for the two processes is obtained by applying a 2:3 ratio between fuel fabrication and reprocessing, obtained from (Platt, 2007), summarized in Table F2/D2-4 of (Shropshire et al., 2009). Table A.5 gives the resulting costs for FR fuel fabrication and UF reprocessing. Table A.5. Supplemental FR cost data

\begin{tabular}{llc} 
Technology & Value & Unit \\
\hline Fuel Fabrication & 2400 & $\$ / \mathrm{kg}$ IHM \\
Reprocessing & 3600 & $\$ / \mathrm{kg}$ IHM
\end{tabular}




\section{REFERENCES}

[ANS, 2009] American Nuclear Society. (2009). Creation of an Independent Entity to Manage U.S. Used Nuclear Fuel (Position Statement 22).

[Arrow, 1999] Arrow, K. J. (1999). Discounting, morality, and gaming. Discounting and intergenerational equity, 13-21.

[Azar et al., 2003] Azar, C., Lindgren, K., \& Andersson, B. A. (2003). Global energy scenarios meeting stringent $\mathrm{CO} 2$ constraints-cost-effective fuel choices in the transportation sector. Energy Policy, 31(10), 961-976.

[Birge and Louveaux, 2011] Birge, J. R., \& Louveaux, F. (2011). Introduction to stochastic programming. Springer.

[Dixon et al., 2008] Dixon, B., Halsey, B., Kim, S., Matthern, G., Piet, S., \& Shropshire, D. (2008). Dynamic systems analysis report for nuclear fuel recycle. Idaho National Laboratory, doi, 10, 963737.

[DOE, 1986] U.S. Department of Energy. (1986). A Multiattribute Utility Analysis of Sites Nominated For Characterization For The First Radioactive-Waste Repository - A Decision-Aiding Methodology (DOE/RW-0074). Washington, DC.

[DOE, 1986a] U.S. Department of Energy. (1986) Recommendation By The Secretary of Energy of Candidate Sites For Site Characterization For The First RadioactiveWaste Repository (DOE/S-0048). Washington, DC.

[DOE, 2002] U.S. Department of Energy. (2002). A Technology Roadmap for Generation IV Nuclear Energy Systems (GIF-002-00). Washington, DC.

[DOE, 2006] U.S. Department of Energy. (2006). Report to Congress: Spent Nuclear Fuel Recycling Program Plan. Washington, DC.

[Draper, 2001] Draper, A. J. (2001). Implicit stochastic optimization with limited foresight for reservoir systems (Doctoral dissertation, UNIVERSITY OF CALIFORNIA DAVIS).

[EIA, 2009] Energy Information Administration. (2009). The National Energy Modeling System: An Overview 2009 (DOE/EIA-0581). Washington, DC. Available from: www.eia.doe.gov/oiaf/aeo/overview/

[EMWG, 2007] Economic Modeling Working Group (EMWG) of the Generation IV International Forum. (2007). Cost Estimating Guidelines for Generation IV Nuclear Energy Systems Revision 4.2 (GIF/EMWG/2007/004). 
[Escudero et al., 1993] Escudero, L. F., Kamesam, P. V., King, A. J., \& Wets, R. J. (1993). Production planning via scenario modelling. Annals of Operations Research, 43(6), 309-335.

[Evans, 1981] Evans, N. (1981). Electricity supply modelling: Theory and case study. Energy Research Group, Department of Physics, Cavendish Laboratory, University of Cambridge.

[Evans, 1984] Evans, N. (1984). The Sizewell decision: a sensitivity analysis. Energy economics, 6(1), 14-20.

[Gilbert and Richels, 1981] Gilbert, R. J., \& Richels, R. G. (1981) Reducing uranium resource uncertainty: Is it worth the cost?. Resources and Energy, 3(1), 13-37.

[Gorenstin et al., 1993] Gorenstin, B. G., Campodónico, N. M., da Costa, J. P., \& Pereira, M. V. F. (1993). Power system expansion planning under uncertainty. Power Systems, IEEE Transactions on, 8(1), 129-136.

[Grayson, 1960] Grayson, D. J. (1960). Decisions under uncertainty: Drilling decisions by oil and gas operators: Harvard Business School. Cambridge, Mass, 402.

[Gregory and Lichtenstein, 1987] Gregory, R., \& Lichtenstein, S. (1987). A Review of the High-Level Nuclear Waste Repository Siting Analysis. Risk Analysis, 7(2), 219223.

[Halter and Dean, 1971] Halter, A. N., \& Dean, G. W. (1971). Decisions under uncertainty, with research applications.

[Hamilton et al., 2012] Hamilton, L. H., Scowcroft, B., Ayers, M. H., Bailey, V. A., Carnesale, A., Domenici, P. V., ... \& Sharp, P. (2012). Blue Ribbon Commission on America's Nuclear Future: Report to the Secretary of Energy. US Department of Energy, Washington, DC.

[Havlíček, 2008] Havlíček, L. (2008). Nuclear Fuel Cycle Evaluation and Real Options. Acta Polytechnica, 48(3).

[Huang et al., 1995] Huang, J. P., Poh, K. L., \& Ang, B. W. (1995). Decision analysis in energy and environmental modeling. Energy, 20(9), 843-855.

[IEA, 2013] International Energy Agency. (2013). World Energy Model Documentation (2013 Version). Available from: www.worldenergyoutlook.org

[Juchau et al., 2010] Juchau, C. A., Dunzik-Gougar, M. L., \& Jacobson, J. J. (2010). Modeling the nuclear fuel cycle. Nuclear technology, 171(2), 136-141. 
[Judd and Weissenberger, 1982] Judd, B. R., \& Weissenberger, S. (1982). A systematic approach to nuclear safeguards decision-making. Management Science, 28(3), 289302.

[Kaufman, 1963] Kaufman, G. M. (1963). Statistical decision and related techniques in oil and gas exploration. Prentice-Hall.

[Keppo et al., 2009] Keppo, I., Strubegger, M., \& Riahi, K. (2009). Implications of limited foresight and sequential decision making for long-term energy system planning: an application of the myopic MESSAGE model. IIASA Interim Report IR-09, 6, 17.

[Kim and Edmonds, 2007] Kim, S. H., \& Edmonds, J. (2007). The Challenges and Potential of Nuclear Energy for Addressing Climate Change. Pacific Northwest National Laboratory, PNNL-17037.

[Kim et al., 2006] Kim, S. H., Edmonds, J., Lurz, J., Smith, S. J., \& Wise, M. (2006). The ObjECTS Framework for Integrated Assessment: Hybrid Modeling of Transportation. Energy Journal, 63-91.

[Kunsch and Teghem, 1987] Kunsch, P. L., \& Teghem Jr, J. (1987). Nuclear fuel cycle optimization using multi-objective stochastic linear programming. European journal of operational research, 31(2), 240-249.

[Leibowicz et al., 2012] Leibowicz, B. D., Roumpani, M., \& Larsen, P. H. (2012). Carbon Emissions Caps and the Impact of a Radical Change in Nuclear Electricity Costs.International Journal of Energy Economics and Policy, 3(1), 60-74.

[Loulou et al., 2004] Loulou, R., Goldstein, G., \& Noble, K. (2004). Documentation for the MARKAL Family of Models. Energy Technology Systems Analysis Programme, 65-73.

[Lund et al., 1999] Lund, J. R., Kirby, K. W., Jenkins, M. W., Draper, A. J., Grimes, P. M., Ward, K. B., ... \& Msangi, S. M. (1999). Integrated economic-engineering analysis of California's future water supply. Department of Agricultural and Resource Economics, Department of Civil and Environmental Engineering, University of California.

[Manne, 1979] Manne, A. S. (1979). ETA-MACRO: A model of energy-economy interactions. Advances in the Economics of Energy and Resources, 2, 205-233.

[Manne and Richels, 1978] Manne, A. S., \& Richels, R. G. (1978). A decision analysis of the US breeder reactor program. Energy, 3(6), 747-767. 
[Martinsen et al., 2006] Martinsen, D., Krey, V., Markewitz, P., \& Vögele, S. (2006). A Time Step Energy Process Model for Germany-Model Structure and Results. Energy Studies Review, 14(1), 2.

[Mulvey and Vladimirou, 1991] Mulvey, J. M., \& Vladimirou, H. (1991). Applying the progressive hedging algorithm to stochastic generalized networks. Annals of Operations Research,31(1), 399-424.

[NEA, 2006] Nuclear Energy Agency Organisation for Economic Co-operation and Development (2006). Advanced Nuclear Fuel Cycles and Radioactive Waste Management (NEA No. 5990).

[NEI, 2013] Nuclear Energy Institute. (2013). More Americans Want New Entity to Manage Used Nuclear Fuel. News Releases. Available from: http://www.nei.org/News-Media/Media-Room/News-Releases/More-AmericansWant-New-Entity-to-Manage-Used-Nucl

[North, 1968] North, D. W. (1968). A tutorial introduction to decision theory. Systems Science and Cybernetics, IEEE Transactions on, 4(3), 200-210.

[Nykvist, 2005] Nykvist, B. (2005). Limited foresight in a linear cost minimisation model of the global energy system. Gotherburg: Master's Thesis in Complex Adaptive Systems, Physical Resource Theory, Chalmers University of Technology.

[Pierpoint, 2011] Pierpoint, L. M. (2011). A decision analysis framework for the US nuclear fuel cycle (Doctoral dissertation, Massachusetts Institute of Technology).

[Piet et al., 2006] Piet, S. J., Matthern, G. E., Jacobson, J. J., Laws, C. T., Cadwallader, L. C., Yacout, A. M., ... \& Bailey, G. (2006). Fuel Cycle Scenario Definition, Evaluation, and Trade-offs. Idaho National Laboratory and the Department of Energy.

[Platt, 2007] Platt's Nuclear Fuel. (2007). Combining LWRs, Fast Reactors and ADS Called Best P\&T Option.

[Rockafellar and Wets, 1991] Rockafellar, R. T., \& Wets, R. J. B. (1991). Scenarios and policy aggregation in optimization under uncertainty. Mathematics of operations research, 16(1), 119-147.

[Rothwell, 2006] Rothwell, G. (2006). A real options approach to evaluating new nuclear power plants. The Energy Journal, 37-53.

[Schneider et al., 2005] Schneider, E. A., Bathke, C. G., \& James, M. R. (2005). NFCSIM: a dynamic fuel burnup and fuel cycle simulation tool. Nuclear technology, 151(1), $35-50$. 
[Shropshire et al., 2009] Shropshire, D. E., Williams, K. A., Smith, J. D., Dixon, B. W., Dunzik-Gougar, M., Adams, R. D., ... \& Hebditch, D. Advanced Fuel Cycle Cost Basis. Idaho National Laboratory report, December, 2009. INL/EXT-07-12107.

[Teghem et al., 1986] Teghem Jr, J., Dufrane, D., Thauvoye, M., \& Kunsch, P. (1986). STRANGE: an interactive method for multi-objective linear programming under uncertainty.European Journal of Operational Research, 26(1), 65-82.

[Van Den Durpel et al., 2003] Van Den Durpel, L., Yacout, A., Wade, D., \& Khalil, H. (2003). DANESS-Dynamic analysis of nuclear energy system strategies. In Proc. of the Global 2003 Conference, New Orleans, LA, USA, November 16-20.

[Wagner et al., 2012] Wagner, J. C., Peterson, J. L., Mueller, D. E., Gehin, J. C., Worrall, A., Taiwo, T., ... \& Carter, J. T. Categorization of Used Nuclear Fuel Inventory in Support of a Comprehensive National Nuclear Fuel Cycle Strategy. Oak Ridge National Laboratory report, December, 2012. ORNL/TM-2012/308, FCRD-FCT2012-000232.

[Wald, 1945] Wald, A. (1945). Statistical decision functions which minimize the maximum risk. Annals of Mathematics, 265-280.

[Weyant and Olavson, 1999] Weyant, J. P., \& Olavson, T. (1999). Issues in modeling induced technological change in energy, environmental, and climate policy. Environmental Modeling \& Assessment, 4(2-3), 67-85.

[Wilson et al., 2012] Wilson, P. P. H., Gidden, M., Huff, K., \& Carlsen, R. (2012). Cyclus: A Nuclear Fuel Cycle Code from the University of Wisconsin Madison. Available from: cyclus.github.com.

[Yacout et al., 2004] Yacout, A. M., Hill, R. N., Van Den Durpel, L., Finck, P. J., Schneider, E. A., Bathke, C. G., \& Herring, J. S. (2004). Dynamic analysis of the AFCI scenarios. Growth, 400000, 500000.

[Yacout et al., 2006] Yacout, A. M., Jacobson, J. J., Matthern, G. E., Piet, S. J., Shropshire, D. E., \& Laws, C. T. (2006). VISION-verifiable fuel cycle simulation of nuclear fuel cycle dynamics. Waste Management.

[Yao and Jaafari, 2003] Yao, J., \& Jaafari, A. (2003). Combining real options and decision tree: an integrative approach for project investment decisions and risk management. The Journal of Structured Finance, 9(3), 53-70.

[Zhou et al., 2006] Zhou, P., Ang, B. W., \& Poh, K. L. (2006). Decision analysis in energy and environmental modeling: An update. Energy, 31(14), 2604-2622. 


\section{VITA}

Urairisa Birdy Phathanapirom was born and raised in Fort Worth, Texas. During her undergraduate career, she mentored a young girls' physics camp, pursued undergraduate research at the Nuclear Engineering Teaching Laboratory, and explored radiochemistry at the University of Nevada, Las Vegas. In 2008, she received a Bachelor's of Science in Physics from the University of Texas at Austin and began graduate work in the Nuclear and Radiation Engineering Program. Her research focuses on fuel cycle analysis, fuel cycle metrics, and decision making in transition analyses. Birdy is a world explorer and adventure seeker.

Permanent email: bphathanapirom@utexas.edu

This thesis was typed by the author. 